\title{
A Test of Momentum Trading Strategies in Foreign Exchange Markets:
}

\section{Evidence from the G7}

Rob Bianchi, Michael E. Drew* and John Polichronis

School of Economics and Finance, Queensland University of Technology, GPO Box 2434, Brisbane, Queensland, Australia, 4000.

\footnotetext{
* Corresponding author: Email: m.drew@qut.edu.au; Tel: +61-7-3864-1481; Fax: +61-7-3864-1500. We gratefully acknowledge the 'Applied Modeling in Economics \& Finance (AMEF)' Research Centre, School of Economics and Finance, Faculty of Business, Queensland University of Technology for financial assistance. Any remaining errors are our own.
} 


\begin{abstract}
In this trading strategy study, we ask three questions. First, does momentum exist in foreign exchange markets? Second, what is the impact of transactions costs on excess returns? And, third, can a consolidated trading signal garner excess returns and, if so, what is the source of such returns? Using total return momentum strategies in the foreign exchange markets of the G7 for the period 1980 through 2004, the answers from this study are as follows: we find evidence of momentum; however, such momentum appears transitory, particularly for longer look back periods. As expected, transaction costs have a material negative impact on excess returns. Finally, a consolidated signal garners excess returns; however, a bootstrap simulation finds the source of these returns is a function of autocorrelation.
\end{abstract}

\title{
JEL Classification: F31, G14
}

Key words: $\quad$ Foreign exchange, momentum, trading rules 


\section{Introduction}

One of the most hotly contested ideas in the study of financial economics relates to the notion that capital markets are efficient in an informational sense. Trading rules, based on the premise that historical data is information rich about the future direction of asset prices, defies the received academic position of the efficient markets hypothesis (EMH) (Fama, 1970). However, one of the empirical challenges with tests of the EMH relates to the ambivalence that researchers have in rejecting the null hypothesis (in turn, providing acceptance to an extreme alternate hypothesis, that is, market inefficiency) and having employed a methodology limited by issues including: data mining, structural change and model instability; and, market volatility. It is our conjecture that it is this combination of an extreme alternate hypothesis (that is, market inefficiency), methodological limitations, and, the chance for profit, that has led to nothing short of a fascination regarding the topic of capital market efficiency by academe and practitioners alike.

While a complete review of the voluminous number of empirical tests of trading rules in capital markets is beyond the scope of this paper, it is appropriate to identify those seminal contributions that provide a rationale for the agenda undertaken in this study. Shiller (2003) contends that up to the end of the 1970s, a naïve strategy of asset selection was supported by the body of theoretical and empirical work in financial economics. However, in the 1980s, Brozynski, Menkhoff, and Schmidt (2003) note that an influential challenge arose in the work of De Bondt and Thaler (1985), which claimed that a contrarian strategy would be profitable over a time period of several years (see also Lakonishok, Shleifer and Vishny, 1994). The 1990s saw the work of Jegadeesh and 
Titman (1993) exploit momentum-based strategies for profit at horizons of around six months. ${ }^{1}$

These ideas, historically tested in stock markets, have also been considered in foreign exchange markets, with various studies attempting to explain the presence of excess returns. Trading rule studies, such as Sweeney (1986), Taylor and Allen (1992) and Brock, Lakonishok and LeBaron (1992) have questioned the notion of market efficiency in foreign exchange markets on the basis of return predictability. Other studies such as Kho (1996) argue that excess returns are the result of time varying risk premia and test for the presence of GARCH processes in foreign exchange returns. A further strand of literature, led by Szakmary and Mathur (1997), consider the role of central bank intervention in markets, suggesting the central banks lack incentives to profit from market fluctuations.

While the source of excess returns is up for debate, the literature overwhelmingly provides corroborating results of the profitability of trading strategies in foreign exchange markets. Important contributions by Sweeney (1986), Taylor and Allen (1992), Levich and Thomas (1993), Kho (1996), Dutt and Ghosh (1999), LeBaron (1999), Marsh (2000) and, into the new century by Okunev and White (2003), have reported excess returns using a variety of ex-ante trading rules, particularly rules based on moving average filters. However, while the foreign exchange literature is voluminous on empirical research that has defined momentum in the form of moving averages (that is, when a trading decision is the result of some form of moving average crossover), there is a paucity of research

\footnotetext{
${ }^{1}$ See also the update by Jegadeesh and Titman (2001).
} 
that considers momentum-based filters as defined by Jegadeesh and Titman (1993), that is, measuring momentum as total returns over a historical formation period.

In this paper, we test the practitioner (and emerging academic) consensus that movements in foreign exchange markets are predictable. In an excellent recent survey of tests of foreign exchange market efficiency, Lewis (1995) demonstrates that the results are, at times, inconsistent and are open to important criticisms in terms of the methodological approach employed. $^{2}$ Lewis (1995) is not alone in this critique, with an important contribution by Neely, Weller and Dittmar (1997) regarding the "narrowness" of the definition of various trading strategies and resultant excess returns reported by empirical studies being open to question. We respond to the methodological challenge in this paper by employing a range of commonly employed momentum strategies (of the form of Jegadeesh and Titman, 1993) used on the dealing desks of foreign exchange traders around the world.

\section{Data Collection}

The dataset employed in this study consisted of the G7 countries (Canada, France, Germany, Italy, Japan, the U.K., and the U.S.) which were sourced from Global Financial Data, Inc. The data consisted of monthly observations from November 1980 to January 2004. Due to the introduction of the Euro currency on 31 December 1998 whereby the German Deutchemark, French Franc and Italian Lira currencies were fixed to the value of the Euro, the analysis in this paper is divided into two time periods. The first time period is from November 1980 through December 1998, consisting of 217 monthly return

\footnotetext{
2 This study is structured as a detailed empirical investigation examining foreign exchange market efficiency using popular momentum strategies currently employed by practitioners. We are motivated in this empirical study to focus on the methodological and results sections of the larger study, and hence we are brief about reviewing the literature to date. For an excellent survey of this area, see Lewis (1995).
} 
observations, and, the second period is from January 1999 through January 2004, consisting of 61 monthly return observations. ${ }^{3}$ The dataset consisted of the monthly spot exchange rates and the three-month interbank rates of each G7 nation.

From this dataset, two types of data series were constructed. The first data series consisted of the spot monthly returns of the G7 countries. These base currency returns of each currency pair were computed as follows:

$$
R_{B, t}=\frac{S_{t}}{S_{t-1}}-1
$$

where $R_{B, t}$ equates to the base currency return, $S_{t}$ is the spot foreign exchange rate at month $t$ and $S_{t-1}$ equates to the spot foreign exchange rate at month $t-1$. The foreign currency spot rate returns for each currency pair of the G7 countries were then calculated. These base currency returns are returns of the domestic (base) currency per unit of foreign currency. Effectively, these calculations are a time series of monthly returns of the fluctuations of each cross rate combination of all G7 currency pairs.

The second data series comprises the first dataset of spot returns, and incorporates the interest rate differential of each currency pair. Effectively, an investor that allocates capital to a foreign currency is not only exposed to fluctuations of the spot rates between the domestic (base) currency and the foreign currency, but the investor is also exposed to

\footnotetext{
${ }^{3}$ The first data period ceases at December 1998 as the German Deutschemark, French Franc and Italian Lira exchange rates were fixed to form the Euro currency on 01 January 1999 at the respective exchange rates of 1 Euro equal to 1.95583 German marks, 6.55957 French Francs and 1,936.27 Italian Lire (Official Journal of the European Communities, 1998).
} 
the interest rate differential during the investment time horizon. As this study analyses monthly returns, we assume that the investor is exposed to the one-month interest rate differential of each currency pair. The returns in the second data series were computed as follows:

$$
R_{I, t}=\left(r_{f}-r_{d}\right) * \frac{1}{12}+\frac{S_{t}}{S_{t-1}}-1
$$

where $R_{I, t}$ equates to the interest adjusted foreign currency monthly return, $r_{f}$ is the one month interest rate of the foreign currency, $r_{d}$ is the one month interest rate of the domestic (i.e. base) currency, and $\left(r_{f}-r_{d}\right) * \frac{1}{12}$ equates to the monthly interest rate differential gain or loss, $S_{t}$ is the spot foreign exchange rate at month $t$ and $S_{t-1}$ equates to the spot foreign exchange rate at month $t-1$.

Considering that Global Financial Data, Inc did not make available the historical onemonth interest rate for each G7 nation, we resorted to utilising the three-month interest rates, and thus, we therefore assumed a flat yield curve in each currency from one month to three months in order to use the three month interest rate as the proxy for the one month interest rate. ${ }^{4}$ For future reference, this second data series is referred to as the “interest-adjusted returns”, representing the actual returns that investors would earn if they converted their base currencies into each foreign currency and held that currency for a one month time horizon.

\footnotetext{
${ }^{4}$ This approach has some standing in the literature; see Okunev and White (2003).
} 
Tables 1 and 2 present the summary statistics for the base currency returns of each country and each respective currency pair. The descriptive statistics from Table 1 indicate that the Japanese yen clearly appreciated across all currencies while the Italian Lira depreciated across all currencies during the 1980 to 1998 period. During this period, the Jarque-Bera statistic indicates that thirty-three out of the forty-two cross rates reject the hypothesis of normally distributed returns. It is clear that the IID assumption is unreasonable when performing an analysis of spot rate returns on the G7 countries during this time period and this finding is consistent with similar findings on weekly currency data in Kho (1996). Table 2 considers the same summary statistics for the time period since the introduction of the Euro currency from January 1999 to January 2004. Contrary to Table 1, the returns in Table 2 do not reject the hypothesis of normally distributed returns with the exception of the Japanese Yen-Euro currency pair.

[Insert Tables 1 and 2 about here]

Similar to the summary statistics presented for base currency returns, Tables 3 and 4 provide the descriptive statistics of the interest-adjusted returns for the 1980 to 1998 and 1999 to 2004 time periods. The evidence provided in Tables 3 and 4 highlight that when the interest rate differential between each currency pair is considered in the total return to the investor, one can see that the losses on spot rate appreciation by Japanese investors are offset by the higher interest rate earned by holding foreign currencies. Conversely, the spot rate currency profits achieved by Italian investors were offset by the negative interest rate differential when holding those foreign currencies. ${ }^{5}$

\footnotetext{
${ }^{5}$ One may interpret this result as being consistent with the theory of interest rate parity or evidence of unbiased expectations. For a more complete discussion of this debate, see Froot and Thaler (1990).
} 
[Insert Tables 3 and 4 about here]

Finally, the data collection process required two sets of returns to be generated. This paper defines momentum similar to Jegadeesh and Titman (1993) by measuring momentum as the total return based on a specified historical formation period. This simple momentum trading rule calculated on base currency returns can be defined as:

$R_{M F B, t-1}=\prod_{n<t}^{n}\left(1+R_{B, n}\right)-1$

where $R_{M F B, t-1}$ is the formation period return which is the total return of a foreign currency for $n$ periods, and $\prod_{n<t}^{n}\left(1+R_{B, n}\right)$ is the sum product of $n$ monthly base currency returns prior to time $t$.

While this methodology measures momentum as the total return derived from historical foreign exchange rates, we extend the definition of momentum by employing the second dataset and defining interest adjusted returns as:

$$
R_{M F I, t-1}=\prod_{n<t}^{n}\left(1+R_{I, n}\right)-1
$$

where $R_{M F I, t-1}$ is the formation period return which is the total return of a foreign currency for $n$ periods and $\prod_{n<t}^{n}\left(1+R_{I, n}\right)$ is the sum product of $n$ monthly interest adjusted 
returns prior to time $t .^{6}$ With issues of data completed, we now explore the methodological approach used in this study.

\section{Methodology}

We take a four-step approach to investigating momentum in this study, comprising (a) calculating momentum returns across various look back periods; (b) examining the role of transaction costs on excess returns; (c) consolidating all look back periods into a single signal to avoid bias; and, (d) bootstrapping of results to evaluate the source of any excess returns. We consider each of these methodological tenets in the following section.

We commence with the development of the naïve trading strategies. When the momentum returns were calculated, each foreign currency was ranked from highest to lowest as defined by the historical momentum return as at the end of each formation month at period $t-1$ with respect to its base currency. The next step in this naïve trading rule was to engage in a series of strategies to create long positions in the foreign currencies with the highest momentum return and create short positions in the foreign currencies with the lowest momentum return. We develop two naïve strategies: (a) go long the foreign currency with the strongest momentum, and, an equal weighted short position in the foreign currency with the weakest momentum; (b) an equal-weighted long position of the two foreign currencies with the strongest momentum, and, an equalweighted short position of the two foreign currencies with the weakest momentum. We

\footnotetext{
${ }^{6}$ The performance of a momentum strategy based on interest adjusted returns is important for three reasons. First, momentum tests defined as arithmetic average returns were evaluated by Sweeney (1986) while Okunev and White (2003) examined multiple moving averages on both spot rates and interest adjusted returns. Our contribution to this debate is that we consider this problem from a different perspective through an alternate specification of momentum which is closer to that of Jegadeesh and Titman (1993). Second, the definition of interest adjusted returns is important in the foreign currency literature as it represents the actual returns earned by investors when they allocate capital into respective foreign currencies. Finally, the momentum strategy employed in this study is simple and can be easily replicated by fund managers and currency traders alike.
} 
calculate the momentum of these two strategies using both data sets, that is, raw spot returns and on interest adjusted returns.

The second methodological step examines the role of transaction costs on excess returns. After the profitability and statistical significance of momentum is evaluated across data sets, we then identified the two most profitable momentum strategies from each base currency and applied transaction costs to them. Transaction costs are applied as a test of the robustness of any findings in terms of applicability in the field.

As the results of any trading strategy study may be transitionary, the third methodological step attempts to avoid such bias. In order to avoid the favoured momentum look-back period or "avoid cherry picking”, this study consolidated all of the momentum rankings from all the various formation periods into one consolidated test. The findings from the consolidated rankings show that statistically significant returns were garnered from the consolidation of the various look-back rankings.

Finally, with the finding of statistical significance, the paper then turns its attention to investigating why such a momentum strategy works in foreign exchange markets. We take a non-parametric approach to momentum profits, employing a conventional bootstrap technique which randomly selects currency returns which inherently avoids the presence of autocorrelation structure within the time series (by avoiding the blockbootstrap approach, any autocorrelation structure in the returns are eliminated from the simulations). We boostrap each momentum period strategy and then re-compute the consolidated strategy, testing the null hypothesis that momentum profits from the 
consolidated strategy are the result of autocorrelation structure in the returns. This study finds that the autocorrelation of returns is the primary source of the excess returns.

\section{Analysis}

\section{A. Preliminary Results}

The results of the two momentum strategies for the G7 currencies over the 1980 to 1998 and 1999 to 2004 time periods are presented in Tables 5 to 8. The analysis measured momentum return as defined as base currency returns (raw spot returns) and interested adjusted returns. The method employed to determine the statistical significance of excess profits from these strategies was to compare these active strategies with a passive buyand-hold foreign currency portfolio that has an equal weighted long position across the respective six alternate foreign currencies. The study employed both the standard $t$-test and the non-parametric Wilcoxon test in order to measure the statistical significance of excess returns.

[Insert Table 5 (Strategy 1 80-98) and Table 6 (Strategy 2 80-98) about here]

The results indicate that the trading strategies that measured momentum using interest adjusted returns seemed to produce higher excess returns than the same strategy using base currency returns as the measure of momentum. It seems that Strategy One tended to generate higher and more statistically significant excess returns than Strategy Two. In addition, it seems that Strategy One seems to be more profitable than Strategy Two. Finally, the results show that excess returns vary across the various formation periods of 1 to 18 months, however, shorter formation periods tend to be more consistently profitable than longer look back formation periods. 
[Insert Table 7 (Strategy 1 99-04) and Table 8 (Strategy 2 99-04) about here]

The results indicate that the 1980 to 1998 time period produced statistically significance excess returns while the 1999 to 2004 time period generated lower and less statistically significance returns. This could be a function of two factors, namely, the excess returns garnered from momentum may be transitory, and, the 1999 to 2004 data series was a short data period consisting of 61 return observations only, thus making statistical inference difficult. The results clearly show that the currencies that produced the most statistically significant excess returns came from the now obsolete German, French and Italian currencies that do not exist anymore.

\section{B. The Impact of Transaction Costs on Excess Returns}

Considering that this study evaluated two trading strategies, tested two sources of momentum, eight look back formation periods and two time periods, this study selected the best two momentum strategies for each currency and estimated the impact of transaction costs. Considering that the original data returns do not conform to the assumption of normality, the method used to select the best strategies was the level of the Wilcoxon test. Table 9 presents the best two trading strategies of each currency in the 1980 to 1998 time period and assesses the impact that transaction costs has on each of these strategies. The results show how the impact of transaction costs erodes the level of excess returns and reduces the level of statistical significance from the original results which contained zero transaction costs. 
[Insert Table 9 around here]

\section{Momentum and its Time-Varying Effects}

The results in Tables 5 through eight clearly show that the profitability of momentum is variable and depends on the currencies selected and the formation look back period selected. Critics of trading rule studies state that the process of data mining or 'cherry picking' that is introduced when selecting the best or worst performing parameter sets brings the results from such studies into question. The criticism from researchers such as Neely, Weller and Dittmar (1997) is valid as a level of bias can be introduced into the empirical research depending on the trading rule selection criteria. In order to avoid this type of bias in this study, we follow the innovative work of Okunev and White (2003) by consolidating the all the momentum rankings of all momentum look back periods in this study into one consolidated test for each currency. This consolidation similar to Okunev and White (2003) is designed to avoid bias as momentum across all the formation time period is employed under this one test. The results are summarised in Table 10.

\section{[Insert Table 10 around here]}

The results in Table 10 show two tests where the rankings from each of the various momentum look back periods were consolidated to form one consolidated ranking set and that new ranking set were back tested. Considering that the individual momentum clearly show little or no excess returns could be garnered from the 15 and 18 month momentum formation periods, this study provides two back tests, where one test include and the other excludes the 15 to 18 month formation period rankings. The results of these back tests persistently show excess returns, which are statistically significant when compared 
to the passive buy-and -hold benchmark currency portfolio. Interestingly, currencies such as the US dollar and Japanese Yen, which generated little or no statistically significant excess returns on various momentum look back periods, have produced significant excess returns when the various momentum rankings are consolidated ${ }^{7}$. Similar to the individual results, the most significant excess returns in the 1980 to 1998 period were generated from the three European currencies that no longer exist. Interestingly, over the 1999 to 2004 period, Table 10 shows that the consolidated ranking back test generates high levels of statistically significant excess returns. The puzzling feature of the 1999-2004 back test is that individual momentum tests did not produce statistically significant excess returns however, when these rankings were consolidated into a full test of all the formation period rankings, the results exhibited in Table 10 shows that the strategies are highly profitable at a nil transaction cost basis.

\section{The Source of Excess Returns}

The source of excess returns in foreign currency markets is a much debated issue with various theories that attempt to explain this market efficiency anomaly. We take a similar approach to Brock et. al., (1992) and employ the Efron (1979) non-parametric bootstrap approach to this problem. In order to measure the importance of historical information to the profitable momentum strategies in this study, we replicate the foreign currency returns in a bootstrap simulation; however, we impose the absence of autocorrelation of returns. The results of the consolidated Strategy One on simulated data is presented in Table 11.

\footnotetext{
7 The USD back test generated a Wilcoxon test of 1.635, which is statistically significant at the $10 \%$ level and just outside the 5\% significance level. All other Wilcoxon tests are significant at the 5\% level.
} 
The simulation results in Table 11 clearly show that the momentum strategies in this study do not generate excess returns on simulated bootstrap data that assumes zero autocorrelation of returns. The results also indicate that excess returns caused by a bias due to the interest rate differential between currencies would have flowed into these simulation results and caused them to generate excess returns. This clearly has not been the case in this analysis. One can conclude that the excess returns garnered from the momentum strategy in this study is caused by the historical information content in the foreign exchange returns of the original data. The standard deviations and information ratios of the various results in this study clearly indicate that this strategy is not risk free. That is, excess returns can be generated; however, the investor must be exposed to volatility of returns in order to achieve this. Such a result conforms to standard finance theory.

\section{Concluding Comments}

We make a number of concluding comments, as distinct from definitive conclusions, to reflect the imperative for ongoing research in this field. First, momentum, as defined by Jegadeesh and Titman (1993), exists in foreign exchange markets. Second, momentum appears to be largely transitory, albeit, skewed towards short look back or formation periods. Third, when evaluating momentum strategies in light of transaction costs, the ability for traders to garner excess returns after fees is diminished - in short, large trading institutions (characterised by the lowest possible transactions costs) may be able to exploit such opportunities, however, corporate and retail clients (characterised by 
relatively high costs) are limited in their ability to achieve such returns. Fourth, when various look back periods are consolidated into a single signal, we find statistically significant excess returns. From a trading perspective, we would discourage the use of single formation periods by traders, suggesting that an all encompassing measure of momentum may mitigate the transitory nature of such profits. Finally, the bootstrap of the currency returns garnered from the consolidated signal clearly shows the presence of memory is required to generate such returns. One area for future research in this field is to develop more advanced definitions of momentum, as this study employed a relatively simple strategy through which to test momentum. The development of such techniques, and the investigation of the sources of potential excess returns resulting from such strategies, is an important issue left to further studies. 


\section{Reference List}

Brock, W., J. Lakonishok, and B. LeBaron, 1992, Simple technical trading rules and the stochastic properties of stock returns, Journal of Finance 47, 1731-1764.

Brozynski, T., L. Menkhoff, and U. Schmidt, 2003, The use of momentum, contrarian and buy-\&-hold strategies: Survey evidence from fund managers, Mimeo, University of Hannover (December).

De Bondt, W. and R. Thaler, 1985, Does the stock market overreact? Journal of Finance 40, 793-808

Dutt, S., and D. Ghosh, 1999, A note on the foreign exchange market efficiency hypothesis, Journal of Economics and Finance 23, 157-161.

Efron, B., 1979, Bootstrap methods: Another look at the jack-knife, The Annals of Statistics 7, 1-26.

Fama, E., 1970, Efficient capital markets, Journal of Finance 25, 383-417

Froot, K., and R. Thaler, 1990, Anomalies: Foreign exchange, Journal of Economic Perspectives 4, 179-192.

Jegadeesh, M., and S. Titman, 1993, Returns to buying winners and selling losers: Implications for stock market efficiency, Journal of Finance 48, 65-91.

Jegadeesh, M., and S. Titman, 2001, Profitability of momentum strategies: An evaluation of alternative explanations, Journal of Finance 56, 699-720.

Kho, B., 1996, Time-varying risk premia, volatility, and technical trading rule profits: Evidence from foreign exchange futures markets, Journal of Financial Economics 41, 249-290.

Lakonishok, J., A. Shleifer, and R. Vishny, 1994, Contrarian investment, extrapolation, and risk, Journal of Finance 49, 1541-1578

LeBaron, B., 1999, Technical trading rule profitability and foreign exchange intervention, Journal of International Economics 49, 125-143

Levich, R., and L. Thomas, 1993, The significance of technical trading-rule profits in the foreign exchange market: A bootstrap approach, Journal of International Money and Finance 12, 451-474

Lewis, K. 1995, Puzzles in international financial markets, in Handbook of International Economics, Vol. 3, edited by G. Grossman and K. Rogoff, (New York: North Holland).

Marsh, I., 2000, High-frequency Markov switching models in the foreign exchange market, Journal of Forecasting 19, 123-134. 
Neeley, C., P. Weller, and R. Dittmar, Is technical analysis profitable in foreign exchange markets? A genetic programming approach, Journal of Financial and Quantitative Analysis 32, 405-426

Official Journal of the European Communities, 1998, Council Regulation (EC) No. 2866/98, L359, Volume 41, December $31^{\text {st }}$.

Okunev, J., and D. White, 2003, Do momentum-based strategies still work in foreign currency markets? Journal of Financial and Quantitative Analysis 38, 425-447.

Shiller, R., 2003, From efficient markets theory to behavioural finance, Journal of Economic Perspectives 17, 83-104

Sweeney, R., 1986, Beating the foreign exchange market, Journal of Finance 41, 163182.

Szakmary, A., and I. Mathur, 1997, Central bank intervention and trading profit rules in foreign exchange markets, Journal of International Money and Finance 16, 513535

Taylor, M., and H. Allen, 1992, The use of technical analysis in the foreign exchange market, Journal of International Money and Finance 11, 304-314 
TABLE 1

Descriptive Statistics (Base Currency Returns)

$\underline{\text { U.S.A. }} \underline{\text { U.K. }} \underline{\text { Canada }} \underline{\text { Germany }} \underline{\text { France }} \underline{\text { Italy }} \underline{\text { Japan }} \underline{\text { Equal }}$

U.S.A.

\begin{tabular}{|c|c|c|c|c|c|c|c|c|}
\hline Mean Ret. (\%) & NA & -0.108 & -0.107 & 0.124 & -0.049 & -0.221 & 0.368 & 0.001 \\
\hline Median Ret. (\%) & NA & -0.233 & -0.075 & 0.123 & 0.119 & -0.072 & -0.127 & 0.040 \\
\hline Std. Dev. (\%) & NA & 3.322 & 1.290 & 3.354 & 3.277 & 3.194 & 3.741 & 2.468 \\
\hline t-Stat. & NA & -0.480 & -1.221 & 0.543 & -0.220 & -1.017 & 1.447 & 0.006 \\
\hline Infor. Ratio & NA & -0.033 & -0.083 & 0.037 & -0.015 & -0.069 & 0.098 & 0.000 \\
\hline Jarque-Bera & NA & $40.212^{* *}$ & $12.265^{* *}$ & 0.128 & 1.495 & $13.945^{* *}$ & $64.520 * *$ & 0.063 \\
\hline \multicolumn{9}{|l|}{ U.K. } \\
\hline Mean Ret. (\%) & 0.219 & NA & 0.102 & 0.264 & 0.093 & -0.075 & 0.530 & 0.189 \\
\hline Median Ret. (\%) & 0.234 & NA & 0.340 & 0.109 & 0.090 & -0.021 & -0.009 & 0.210 \\
\hline Std. Dev. (\%) & 3.349 & NA & 3.317 & 2.590 & 2.568 & 2.592 & 3.713 & 2.335 \\
\hline t-Stat. & 0.963 & NA & 0.455 & 1.503 & 0.535 & -0.429 & $2.102 *$ & 1.192 \\
\hline Infor. Ratio & 0.065 & NA & 0.031 & 0.102 & 0.036 & -0.029 & 0.143 & 0.081 \\
\hline Jarque-Bera & $47.331^{* *}$ & NA & $45.218^{* *}$ & $20.116^{* *}$ & $24.578 * *$ & 3.635 & $216.449 * *$ & $47.600 * *$ \\
\hline \multicolumn{9}{|l|}{ Canada } \\
\hline Mean Ret. (\%) & 0.124 & 0.006 & NA & 0.242 & 0.068 & -0.105 & 0.488 & 0.137 \\
\hline Median Ret. (\%) & 0.075 & -0.339 & NA & -0.023 & 0.061 & -0.224 & -0.091 & -0.028 \\
\hline Std. Dev. (\%) & 1.299 & 3.290 & NA & 3.431 & 3.345 & 3.207 & 3.864 & 2.534 \\
\hline t-Stat. & 1.403 & 0.027 & NA & 1.037 & 0.301 & -0.484 & 1.860 & 0.797 \\
\hline Infor. Ratio & 0.095 & 0.002 & NA & 0.070 & 0.020 & -0.033 & 0.126 & 0.054 \\
\hline Jarque-Bera & $17.578^{* *}$ & $22.701^{* *}$ & NA & 0.228 & 0.326 & 1.370 & $71.261^{* *}$ & 0.267 \\
\hline \multicolumn{9}{|l|}{ Germany } \\
\hline Mean Ret. (\%) & -0.011 & -0.198 & -0.124 & NA & -0.166 & -0.323 & 0.285 & -0.089 \\
\hline Median Ret. (\%) & -0.123 & -0.109 & 0.023 & NA & -0.039 & -0.104 & -0.090 & 0.011 \\
\hline Std. Dev. (\%) & 3.366 & 2.560 & 3.440 & NA & 0.904 & 1.804 & 3.292 & 1.822 \\
\hline t-Stat. & -0.050 & -1.138 & -0.531 & NA & $-2.703 * *$ & $-2.634 * *$ & 1.273 & -0.723 \\
\hline Infor. Ratio & -0.003 & -0.077 & -0.036 & NA & -0.184 & -0.179 & 0.086 & -0.049 \\
\hline Jarque-Bera & 2.803 & $9.200 * *$ & 3.416 & NA & $2742.385^{* *}$ & $1987.114 * *$ & $95.045^{* *}$ & 0.740 \\
\hline \multicolumn{9}{|l|}{ France } \\
\hline Mean Ret. (\%) & 0.157 & -0.028 & 0.043 & 0.175 & NA & -0.154 & 0.455 & 0.108 \\
\hline Median Ret. (\%) & -0.119 & -0.090 & -0.061 & 0.039 & NA & -0.046 & 0.098 & 0.019 \\
\hline Std. Dev. (\%) & 3.312 & 2.557 & 3.366 & 0.935 & NA & 1.717 & 3.293 & 1.756 \\
\hline t-Stat. & 0.698 & -0.161 & 0.190 & $2.752^{* *}$ & NA & -1.324 & $2.037 *$ & 0.906 \\
\hline Infor. Ratio & 0.047 & -0.011 & 0.013 & 0.187 & NA & -0.090 & 0.138 & 0.062 \\
\hline Jarque-Bera & $7.475 *$ & $14.040 * *$ & 3.070 & $3331.289 * *$ & NA & $3639.509 * *$ & $78.863 * *$ & $18.889 * *$ \\
\hline
\end{tabular}




\section{Italy}

\begin{tabular}{|c|c|c|c|c|c|c|c|c|}
\hline No. of Obs. & 217 & 217 & 217 & 217 & 217 & NA & 217 & 217 \\
\hline Mean Ret. (\%) & 0.325 & 0.143 & 0.209 & $0.358 * *$ & 0.186 & NA & $0.638 *$ & $0.310 *$ \\
\hline Median Ret. (\%) & 0.072 & 0.021 & 0.225 & 0.104 & 0.046 & NA & 0.148 & 0.180 \\
\hline Std. Dev. (\%) & 3.267 & 2.607 & 3.245 & 1.904 & 1.818 & NA & 3.678 & 1.988 \\
\hline t-Stat. & 1.465 & 0.807 & 0.949 & $2.768^{* *}$ & 1.504 & NA & $2.555^{*}$ & $2.295^{*}$ \\
\hline Infor. Ratio & 0.099 & 0.055 & 0.064 & 0.188 & 0.102 & NA & 0.173 & 0.156 \\
\hline Jarque-Bera & $47.856^{* *}$ & $6.103^{*}$ & $6.980 *$ & $3640.267 * *$ & $6717.516^{* *}$ & NA & $265.174^{* *}$ & $800.425^{* *}$ \\
\hline \multicolumn{9}{|l|}{ Japan } \\
\hline Mean Ret. (\%) & -0.232 & -0.397 & -0.343 & -0.179 & -0.349 & $-0.507^{*}$ & NA & -0.335 \\
\hline Median Ret. (\%) & 0.127 & 0.009 & 0.091 & 0.090 & -0.098 & -0.148 & NA & -0.044 \\
\hline Std. Dev. (\%) & 3.621 & 3.539 & 3.740 & 3.198 & 3.195 & 3.481 & NA & 2.974 \\
\hline t-Stat. & -0.944 & -1.653 & -1.350 & -0.827 & -1.610 & $-2.148 *$ & NA & -1.658 \\
\hline Infor. Ratio & -0.064 & -0.112 & -0.092 & -0.056 & -0.109 & -0.146 & NA & -0.113 \\
\hline Jarque-Bera & $22.341 * *$ & $89.713 * *$ & $24.992 * *$ & $32.351^{* *}$ & $25.589 * *$ & $107.728 * *$ & NA & $94.437 * *$ \\
\hline
\end{tabular}

The dataset consists of monthly returns of individual G7 currency pairs from November 1980 to December 1998.

The time period consists of 217 monthly return observations. The Equal column is the currency return based on an Equal weighting allocated to the six respective foreign currencies. The Jarque-Bera test of normality is based on Skewness and excess kurtosis and is Chi-square distributed with two degrees of freedom.

Statistical significance at the $1 \%$ level and $5 \%$ level is denoted by ** and *, respectively. 
TABLE 2

Descriptive Statistics (Base Currency Returns)

\begin{tabular}{|c|c|c|c|c|c|c|c|}
\hline & U.S.A. & $\underline{\text { U.K. }}$ & Canada & $\underline{\text { Euro }}$ & $\underline{\text { Japan }}$ & $\underline{\text { Equal } \wedge}$ & $\underline{\text { Equal } \wedge \wedge}$ \\
\hline \multicolumn{8}{|l|}{ U.S.A. } \\
\hline Mean Ret. (\%) & NA & 0.185 & 0.254 & 0.144 & 0.153 & 0.171 & 0.184 \\
\hline Median Ret. (\%) & NA & -0.093 & 0.166 & -0.345 & -0.088 & -0.153 & -0.002 \\
\hline Std. Dev. (\%) & NA & 2.228 & 1.857 & 3.020 & 2.888 & 2.134 & 1.809 \\
\hline t-Stat. & NA & 0.649 & 1.067 & 0.373 & 0.413 & 0.624 & 0.794 \\
\hline Infor. Ratio & NA & 0.083 & 0.137 & 0.048 & 0.053 & 0.080 & 0.102 \\
\hline Jarque-Bera & NA & 1.042 & 0.220 & 2.465 & 0.039 & 1.580 & 0.515 \\
\hline \multicolumn{8}{|l|}{ U.K. } \\
\hline Mean Ret. (\%) & -0.137 & NA & 0.109 & -0.038 & -0.002 & -0.024 & -0.017 \\
\hline Median Ret. (\%) & 0.093 & NA & 0.368 & -0.012 & -0.371 & -0.057 & -0.001 \\
\hline Std. Dev. (\%) & 2.210 & NA & 2.607 & 2.185 & 3.102 & 1.720 & 1.842 \\
\hline t-Stat. & -0.483 & NA & 0.327 & -0.135 & -0.005 & -0.108 & -0.071 \\
\hline Infor. Ratio & -0.062 & NA & 0.042 & -0.017 & -0.001 & -0.014 & -0.009 \\
\hline Jarque-Bera & 0.749 & NA & 0.274 & 2.164 & 0.518 & 7.935 & 2.975 \\
\hline \multicolumn{8}{|l|}{ Canada } \\
\hline Mean Ret. (\%) & -0.219 & -0.042 & NA & -0.094 & -0.077 & -0.103 & -0.108 \\
\hline Median Ret. (\%) & -0.166 & -0.366 & NA & -0.559 & -0.329 & -0.688 & -0.415 \\
\hline Std. Dev. (\%) & 1.854 & 2.619 & NA & 2.960 & 3.138 & 2.243 & 2.059 \\
\hline t-Stat. & -0.924 & -0.125 & NA & -0.247 & -0.191 & -0.360 & -0.409 \\
\hline Infor. Ratio & -0.118 & -0.016 & NA & -0.032 & -0.024 & -0.046 & -0.052 \\
\hline Jarque-Bera & 0.561 & 1.000 & NA & 2.410 & 1.505 & 3.731 & 4.940 \\
\hline \multicolumn{8}{|l|}{ Euro } \\
\hline Mean Ret. (\%) & -0.053 & 0.087 & 0.182 & NA & 0.069 & 0.046 & 0.023 \\
\hline Median Ret. (\%) & 0.336 & -0.007 & 0.562 & NA & -0.157 & 0.080 & 0.068 \\
\hline Std. Dev. (\%) & 2.983 & 2.175 & 2.944 & NA & 3.346 & 1.588 & 1.791 \\
\hline t-Stat. & -0.140 & 0.311 & 0.482 & NA & 0.161 & 0.225 & 0.102 \\
\hline Infor. Ratio & -0.018 & 0.040 & 0.062 & NA & 0.021 & 0.029 & 0.013 \\
\hline Jarque-Bera & 1.626 & 1.202 & 1.905 & NA & 5.720 & 0.385 & 0.502 \\
\hline \multicolumn{8}{|l|}{ Japan } \\
\hline Mean Ret. (\%) & -0.070 & 0.097 & 0.173 & 0.043 & NA & 0.055 & 0.028 \\
\hline Median Ret. (\%) & 0.088 & 0.372 & 0.330 & 0.160 & NA & 0.172 & 0.008 \\
\hline Std. Dev. (\%) & 2.893 & 3.122 & 3.119 & 3.349 & NA & 2.821 & 2.846 \\
\hline t-Stat. & -0.190 & 0.243 & 0.433 & 0.101 & NA & 0.152 & 0.077 \\
\hline Infor. Ratio & -0.024 & 0.031 & 0.055 & 0.013 & NA & 0.019 & 0.010 \\
\hline Jarque-Bera & 0.602 & 2.160 & 0.348 & $10.079 * *$ & NA & 4.588 & 4.695 \\
\hline
\end{tabular}

The dataset consists of monthly returns of individual G7 currency pairs from January 1999 to January 2004. The time period consists of 61 monthly return observations. The Equal^ column is the currency return based on an equal weighting allocated to the six respective foreign currencies whereby the Euro represents the currencies of Germany, France and Italy The Equal M column is the currency return based on an equal weighting allocated to four respective foreign currencies whereby the Euro represents one currency only The Jarque-Bera test of normality is based on skewness and excess kurtosis and is Chi-square distributed with two degrees of freedom. Statistical significance at the $1 \%$ level and $5 \%$ level is denoted by ** and *, respectively. 
TABLE 3

Descriptive Statistics (Interest Adjusted Currency Returns)

$\underline{\text { U.S.A. }} \underline{\text { U.K. }} \underline{\text { Canada }} \underline{\text { Germany }} \underline{\text { France }} \underline{\text { Italy }} \underline{\text { Japan }}$

U.S.A.

\begin{tabular}{|c|c|c|c|c|c|c|c|c|}
\hline Mean Ret. (\%) & NA & 0.117 & 0.067 & 0.040 & 0.133 & 0.247 & 0.131 & 0.122 \\
\hline Median Ret. (\%) & NA & -0.029 & 0.101 & 0.127 & 0.350 & 0.531 & -0.332 & 0.128 \\
\hline Std. Dev. (\%) & NA & 3.366 & 1.315 & 3.383 & 3.299 & 3.184 & 3.785 & 2.495 \\
\hline t-Stat. & NA & 0.511 & 0.749 & 0.176 & 0.592 & 1.143 & 0.508 & 0.722 \\
\hline Infor. Ratio & NA & 0.035 & 0.051 & 0.012 & 0.040 & 0.078 & 0.035 & 0.049 \\
\hline Jarque-Bera & NA & $34.594 * *$ & $6.270 *$ & 0.076 & 1.121 & $8.253 *$ & $53.279 * *$ & 0.074 \\
\hline \multicolumn{9}{|l|}{ U.K. } \\
\hline Mean Ret. (\%) & -0.006 & NA & 0.051 & -0.044 & 0.050 & 0.167 & 0.068 & 0.048 \\
\hline Median Ret. (\%) & 0.033 & NA & 0.233 & -0.162 & 0.059 & 0.226 & -0.525 & 0.139 \\
\hline Std. Dev. (\%) & 3.391 & NA & 3.365 & 2.600 & 2.589 & 2.600 & 3.740 & 2.365 \\
\hline t-Stat. & -0.026 & NA & 0.225 & -0.248 & 0.285 & 0.949 & 0.268 & 0.298 \\
\hline Infor. Ratio & -0.002 & NA & 0.015 & -0.017 & 0.019 & 0.064 & 0.018 & 0.020 \\
\hline Jarque-Bera & $38.632 * *$ & NA & $38.805^{* *}$ & $23.425^{* *}$ & $27.585^{* *}$ & 4.504 & $203.649 * *$ & $51.897 * *$ \\
\hline \multicolumn{9}{|l|}{ Canada } \\
\hline Mean Ret. (\%) & -0.050 & 0.057 & NA & -0.015 & 0.076 & 0.189 & 0.077 & 0.056 \\
\hline Median Ret. (\%) & -0.101 & -0.230 & NA & -0.276 & -0.058 & 0.042 & -0.414 & -0.104 \\
\hline Std. Dev. (\%) & 1.323 & 3.338 & NA & 3.456 & 3.360 & 3.199 & 3.898 & 2.556 \\
\hline t-Stat. & -0.557 & 0.252 & NA & -0.065 & 0.334 & 0.868 & 0.291 & 0.321 \\
\hline Infor. Ratio & -0.038 & 0.017 & NA & -0.004 & 0.023 & 0.059 & 0.020 & 0.022 \\
\hline Jarque-Bera & $9.650 * *$ & $19.281^{* *}$ & NA & 0.215 & 0.241 & 0.974 & $63.856^{* *}$ & 0.158 \\
\hline \multicolumn{9}{|l|}{ Germany } \\
\hline Mean Ret. (\%) & 0.072 & 0.110 & 0.133 & NA & 0.099 & 0.228 & 0.131 & 0.129 \\
\hline Median Ret. (\%) & -0.127 & 0.162 & 0.277 & NA & 0.189 & 0.367 & -0.236 & 0.233 \\
\hline Std. Dev. (\%) & 3.395 & 2.570 & 3.465 & NA & 0.884 & 1.796 & 3.303 & 1.830 \\
\hline t-Stat. & 0.311 & 0.631 & 0.565 & NA & 1.644 & 1.872 & 0.583 & 1.036 \\
\hline Infor. Ratio & 0.021 & 0.043 & 0.038 & NA & 0.112 & 0.127 & 0.040 & 0.070 \\
\hline Jarque-Bera & 2.354 & $11.660 * *$ & 3.072 & NA & $2000.816^{* *}$ & $1815.318^{* *}$ & $87.697 * *$ & 0.810 \\
\hline \multicolumn{9}{|l|}{ France } \\
\hline Mean Ret. (\%) & -0.025 & 0.015 & 0.036 & -0.090 & NA & 0.132 & 0.037 & 0.018 \\
\hline Median Ret. (\%) & -0.348 & -0.059 & 0.058 & -0.189 & NA & 0.219 & -0.268 & -0.105 \\
\hline Std. Dev. (\%) & 3.334 & 2.577 & 3.379 & 0.914 & NA & 1.722 & 3.285 & 1.755 \\
\hline t-Stat. & -0.109 & 0.087 & 0.156 & -1.450 & NA & 1.128 & 0.166 & 0.147 \\
\hline Infor. Ratio & -0.007 & 0.006 & 0.011 & -0.098 & NA & 0.077 & 0.011 & 0.010 \\
\hline Jarque-Bera & $6.324 *$ & $14.826^{* *}$ & 2.820 & $2502.609 * *$ & NA & $3015.767 * *$ & $86.683^{* *}$ & $17.059 * *$ \\
\hline
\end{tabular}




$\begin{array}{lcccccccc}\text { Italy } & & & & & & \\ \text { Mean Ret. (\%) } & -0.143 & -0.100 & -0.085 & -0.193 & -0.101 & \text { NA } & -0.067 & -0.115 \\ \text { Median Ret. (\%) } & -0.531 & -0.226 & -0.041 & -0.367 & -0.219 & \text { NA } & -0.532 & -0.230 \\ \text { Std. Dev. (\%) } & 3.254 & 2.613 & 3.235 & 1.895 & 1.820 & \text { NA } & 3.659 & 1.961 \\ \text { t-Stat. } & -0.646 & -0.565 & -0.386 & -1.500 & -0.814 & \text { NA } & -0.269 & -0.861 \\ \text { Infor. Ratio } & -0.044 & -0.038 & -0.026 & -0.102 & -0.055 & \text { NA } & -0.018 & -0.058 \\ \text { Jarque-Bera } & 32.892^{* *} & 5.744 & 5.736 & 3379.474 * * & 5723.731^{* *} & \text { NA } & 262.784^{* *} & 675.382^{* *} \\ \text { Japan } & & & & & & & & \\ \text { Mean Ret. (\%) } & 0.005 & 0.065 & 0.068 & -0.026 & 0.069 & 0.197 & \text { NA } & 0.063 \\ \text { Median Ret. (\%) } & 0.332 & 0.525 & 0.415 & 0.236 & 0.268 & 0.532 & \text { NA } & 0.305 \\ \text { Std. Dev. (\%) } & 3.667 & 3.568 & 3.776 & 3.210 & 3.186 & 3.462 & \text { NA } & 2.988 \\ \text { t-Stat. } & 0.019 & 0.266 & 0.265 & -0.118 & 0.320 & 0.839 & \text { NA } & 0.311 \\ \text { Infor. Ratio } & 0.001 & 0.018 & 0.018 & -0.008 & 0.022 & 0.057 & \text { NA } & 0.021 \\ \text { Jarque-Bera } & 17.373^{* *} & 84.497 * * & 21.513^{* *} & 28.944 * * & 28.901 * * & 107.433^{* *} & \text { NA } & 89.498^{* *} \\ & & & & & & & & \end{array}$

The dataset consists of monthly returns of individual G7 currency pairs from November 1980 to December 1998. The time period consists of 217 monthly return observations. The Equal column is the currency return based on an equal weighting allocated to the six respective foreign currencies. The Jarque-Bera test of normality is based on skewness and excess kurtosis and is Chi-square distributed with two degrees of freedom.

Statistical significance at the $1 \%$ level and $5 \%$ level is denoted by ** and *, respectively. 
TABLE 4

Descriptive Statistics (Interest Adjusted Currency Returns)

\begin{tabular}{|c|c|c|c|c|c|c|c|}
\hline & U.S.A. & $\underline{\text { U.K. }}$ & Canada & Euro & Japan & $\underline{\text { Equal} \wedge}$ & Equal`^ \\
\hline \multicolumn{8}{|l|}{ U.S.A. } \\
\hline Mean Ret. (\%) & NA & 0.313 & 0.303 & 0.134 & -0.112 & 0.156 & 0.162 \\
\hline Median Ret. (\%) & NA & -0.011 & 0.197 & -0.287 & -0.382 & -0.106 & 0.047 \\
\hline Std. Dev. (\%) & NA & 2.258 & 1.876 & 3.064 & 2.912 & 2.173 & 1.847 \\
\hline t-Stat. & NA & 1.081 & 1.261 & 0.341 & -0.301 & 0.562 & 0.683 \\
\hline Infor. Ratio & NA & 0.138 & 0.161 & 0.044 & -0.038 & 0.072 & 0.087 \\
\hline Jarque-Bera & NA & 1.139 & 0.165 & 2.353 & 0.089 & 1.518 & 0.521 \\
\hline \multicolumn{8}{|l|}{ U.K. } \\
\hline Mean Ret. (\%) & -0.264 & NA & 0.031 & -0.175 & -0.394 & -0.187 & -0.198 \\
\hline Median Ret. (\%) & 0.012 & NA & 0.320 & -0.105 & -0.734 & -0.242 & -0.174 \\
\hline Std. Dev. (\%) & 2.240 & NA & 2.616 & 2.203 & 3.100 & 1.725 & 1.847 \\
\hline t-Stat. & -0.920 & NA & 0.093 & -0.622 & -0.992 & -0.845 & -0.839 \\
\hline Infor. Ratio & -0.118 & NA & 0.012 & -0.080 & -0.127 & -0.108 & -0.107 \\
\hline Jarque-Bera & 0.873 & NA & 0.279 & 2.040 & 0.540 & $7.752 *$ & 2.957 \\
\hline \multicolumn{8}{|l|}{ Canada } \\
\hline Mean Ret. (\%) & -0.269 & 0.036 & NA & -0.153 & -0.391 & -0.175 & -0.192 \\
\hline Median Ret. (\%) & -0.196 & -0.319 & NA & -0.688 & -0.663 & -0.663 & -0.526 \\
\hline Std. Dev. (\%) & 1.873 & 2.628 & NA & 2.984 & 3.141 & 2.255 & 2.067 \\
\hline t-Stat. & -1.120 & 0.107 & NA & -0.402 & -0.972 & -0.607 & -0.725 \\
\hline Infor. Ratio & -0.143 & 0.014 & NA & -0.051 & -0.124 & -0.078 & -0.093 \\
\hline Jarque-Bera & 0.389 & 1.011 & NA & 2.389 & 1.290 & 3.584 & 4.737 \\
\hline \multicolumn{8}{|l|}{ Euro } \\
\hline Mean Ret. (\%) & -0.045 & 0.222 & 0.239 & NA & -0.187 & 0.033 & 0.048 \\
\hline Median Ret. (\%) & 0.287 & 0.105 & 0.693 & NA & -0.440 & 0.014 & 0.018 \\
\hline Std. Dev. (\%) & 3.022 & 2.187 & 2.964 & NA & 3.345 & 1.606 & 2.406 \\
\hline t-Stat. & -0.117 & 0.794 & 0.631 & NA & -0.438 & 0.161 & 0.156 \\
\hline Infor. Ratio & -0.015 & 0.102 & 0.081 & NA & -0.056 & 0.021 & 0.020 \\
\hline Jarque-Bera & 1.610 & 1.187 & 1.934 & NA & 5.568 & 0.421 & 0.425 \\
\hline \multicolumn{8}{|l|}{ Japan } \\
\hline Mean Ret. (\%) & 0.194 & 0.489 & 0.487 & 0.297 & NA & 0.349 & 0.369 \\
\hline Median Ret. (\%) & 0.382 & 0.736 & 0.664 & 0.440 & NA & 0.489 & -0.066 \\
\hline Std. Dev. (\%) & 2.919 & 3.120 & 3.123 & 3.351 & NA & 2.820 & 2.725 \\
\hline t-Stat. & 0.520 & 1.224 & 1.218 & 0.693 & NA & 0.967 & 1.058 \\
\hline Infor. Ratio & 0.067 & 0.157 & 0.156 & 0.089 & NA & 0.124 & 0.135 \\
\hline Jarque-Bera & 0.757 & 2.257 & 0.288 & $10.243 * *$ & NA & 4.798 & 1.678 \\
\hline
\end{tabular}

The dataset consists of monthly returns of individual G7 currency pairs from January 1999 to January 2004. The time period consists of 61 monthly return observations. The Equal^ column is the currency return based on an equal weighting allocated to the six respective foreign currencies whereby the Euro represents the currencies of Germany, France and Italy The Equal M column is the currency return based on an equal weighting allocated to four respective foreign currencies whereby the Euro represents one currency only The Jarque-Bera test of normality is based on skewness and excess kurtosis and is Chi-square distributed with two degrees of freedom. Statistical significance at the $1 \%$ level and $5 \%$ level is denoted by ** and *, respectively. 
TABLE 5

Strategy One Results - 1980 to 1998

Panel A: Source of Momentum - Raw Currency Returns

\section{Momentum Formation Period (in months):}

CANADA Mean Ret.(\%) - Active Strategy

Mean Ret.(\%) - Passive Equal Weighted Portfolio

Excess Return (\%)

Std. Dev.(\%) - Active Strategy

Std. Dev.(\%) - Passive Equal Weighted Portfolio Infor. Ratio

Prob > Passive Equal Weighted Portfolio (\%)

: paired t-test

: Wilcoxon test

U.K. $\quad$ Mean Ret.(\%) - Active Strategy

Excess Return (\%)

Std. Dev.(\%) - Active Strategy

Std. Dev.(\%) - Passive Equal Weighted Portfolio

Infor. Ratio

Prob > Passive Equal Weighted Portfolio (\%)

: paired t-test

: Wilcoxon test
Mean Ret.(\%) - Passive Equal Weighted Portfolio

\section{1}

2

3

6

9

12

15

18

\begin{tabular}{cccccccc}
0.531 & 0.516 & 0.485 & 0.264 & 0.425 & 0.217 & 0.037 & -0.141 \\
0.055 & 0.077 & 0.086 & 0.127 & 0.160 & 0.152 & 0.168 & 0.171 \\
0.476 & 0.439 & 0.399 & 0.137 & 0.265 & 0.065 & -0.131 & -0.312 \\
3.259 & 3.412 & 3.471 & 3.709 & 3.713 & 3.569 & 3.643 & 3.792 \\
2.561 & 2.547 & 2.550 & 2.543 & 2.543 & 2.559 & 2.572 & 2.580 \\
0.109 & 0.103 & 0.092 & 0.031 & 0.059 & 0.015 & -0.030 & -0.069 \\
$56.02 \%$ & $54.88 \%$ & $55.61 \%$ & $56.40 \%$ & $60.58 \%$ & $57.07 \%$ & $51.98 \%$ & $50.75 \%$ \\
1.607 & 1.513 & 1.352 & 0.451 & 0.845 & 0.214 & -0.423 & -0.974 \\
$1.974 *$ & 1.621 & 1.820 & 1.111 & 1.502 & 0.886 & 0.119 & -0.640 \\
\hline
\end{tabular}

\begin{tabular}{cccccccc}
0.398 & 0.392 & 0.482 & 0.490 & 0.730 & 0.506 & 0.104 & 0.048 \\
0.055 & 0.068 & 0.039 & 0.039 & -0.008 & 0.002 & -0.002 & -0.007 \\
0.343 & 0.324 & 0.443 & 0.451 & $0.738^{* *}$ & 0.504 & 0.107 & 0.055 \\
3.248 & 3.298 & 3.283 & 3.425 & 3.358 & 3.604 & 3.647 & 3.430 \\
2.368 & 2.366 & 2.334 & 2.345 & 2.315 & 2.296 & 2.311 & 2.325 \\
0.090 & 0.081 & 0.113 & 0.111 & 0.183 & 0.116 & 0.024 & 0.013 \\
$52.31 \%$ & $52.09 \%$ & $50.47 \%$ & $54.03 \%$ & $57.21 \%$ & $56.10 \%$ & $50.99 \%$ & $47.74 \%$ \\
1.330 & 1.187 & 1.647 & 1.617 & $2.644^{* *}$ & 1.666 & 0.341 & 0.183 \\
$1.759 *$ & 1.40 & $1.973^{*}$ & $1.841^{*}$ & $3.105^{* *}$ & $2.550^{* *}$ & 1.060 & 0.323 \\
\hline
\end{tabular}

U.S.A. Mean Ret.(\%) - Active Strategy

Mean Ret.(\%) - Passive Equal Weighted Portfolio

Excess Return (\%)

Std. Dev.(\%) - Active Strategy

Std. Dev.(\%) - Passive Equal Weighted Portfolio

Infor. Ratio

Prob > Passive Equal Weighted Portfolio (\%)

: paired t-test

: Wilcoxon test

JAPAN Mean Ret.(\%) - Active Strategy

Mean Ret.(\%) - Passive Equal Weighted Portfolio

Excess Return (\%)

Std. Dev.(\%) - Active Strategy

Std. Dev.(\%) - Passive Equal Weighted Portfolio

Infor. Ratio

Prob > Passive Equal Weighted Portfolio (\%)

: paired t-test

: Wilcoxon test

\begin{tabular}{cccccccc}
0.269 & 0.351 & 0.485 & 0.410 & 0.517 & 0.130 & 0.046 & 0.028 \\
0.122 & 0.144 & 0.155 & 0.193 & 0.219 & 0.192 & 0.230 & 0.237 \\
0.147 & 0.207 & 0.330 & 0.217 & 0.298 & -0.062 & -0.185 & -0.209 \\
3.216 & 3.288 & 3.509 & 3.399 & 3.353 & 3.534 & 3.525 & 3.393 \\
2.501 & 2.486 & 2.487 & 2.474 & 2.468 & 2.473 & 2.468 & 2.473 \\
0.035 & 0.050 & 0.076 & 0.053 & 0.071 & -0.014 & -0.042 & -0.051 \\
$48.6 \%$ & $45.6 \%$ & $51.4 \%$ & $49.8 \%$ & $54.8 \%$ & $53.7 \%$ & $49.0 \%$ & $45.2 \%$ \\
0.511 & 0.735 & 1.117 & 0.773 & 1.023 & -0.202 & -0.598 & -0.717 \\
0.735 & 0.94 & 1.251 & 1.178 & 1.538 & 0.544 & -0.130 & -0.533 \\
\hline
\end{tabular}

$\begin{array}{llllllll}0.339 & 0.471 & 0.380 & 0.309 & 0.408 & 0.330 & 0.022 & -0.130\end{array}$

$\begin{array}{lllllllll}0.092 & 0.101 & 0.100 & 0.090 & 0.085 & 0.088 & 0.056 & 0.035\end{array}$

$\begin{array}{lllllllll}0.247 & 0.370 & 0.279 & 0.220 & 0.322 & 0.243 & -0.034 & -0.165\end{array}$

$\begin{array}{lllllllll}3.019 & 3.132 & 3.269 & 3.337 & 3.383 & 3.267 & 3.408 & 3.333\end{array}$

$\begin{array}{lllllllll}2.963 & 2.967 & 2.974 & 2.993 & 2.996 & 2.978 & 2.986 & 2.981\end{array}$

$\begin{array}{llllllll}0.056 & 0.083 & 0.061 & 0.047 & 0.068 & 0.052 & -0.007 & -0.035\end{array}$

$\begin{array}{llllllll}46.76 \% & 48.84 \% & 47.66 \% & 50.71 \% & 51.44 \% & 51.22 \% & 48.51 \% & 48.24 \%\end{array}$

$\begin{array}{lllllllll}0.827 & 1.215 & 0.894 & 0.689 & 0.984 & 0.748 & -0.101 & -0.495\end{array}$

\begin{tabular}{llllllll}
0.306 & 0.79 & 0.659 & 0.91 & 1.329 & 1.079 & 0.009 & -0.481 \\
\hline
\end{tabular} 


\begin{tabular}{|c|c|c|c|c|c|c|c|c|c|}
\hline \multirow[t]{9}{*}{ GERMANY } & Mean Ret.(\%) - Active Strategy & 0.526 & 0.350 & 0.493 & 0.470 & 0.621 & 0.375 & 0.063 & 0.071 \\
\hline & Mean Ret.(\%) - Passive Equal Weighted Portfolio & 0.112 & 0.089 & 0.098 & 0.080 & 0.073 & 0.098 & 0.089 & 0.099 \\
\hline & Excess Return (\%) & 0.413 & 0.261 & 0.395 & 0.390 & $0.548^{*}$ & 0.276 & -0.026 & -0.028 \\
\hline & Std. Dev.(\%) - Active Strategy & 3.221 & 3.510 & 3.593 & 3.522 & 3.478 & 3.602 & 3.691 & 3.522 \\
\hline & Std. Dev.(\%) - Passive Equal Weighted Portfolio & 1.818 & 1.790 & 1.789 & 1.787 & 1.799 & 1.775 & 1.784 & 1.794 \\
\hline & Infor. Ratio & 0.117 & 0.066 & 0.099 & 0.095 & 0.138 & 0.066 & -0.006 & -0.007 \\
\hline & Prob > Passive Equal Weighted Portfolio (\%) & $50.5 \%$ & $48.4 \%$ & $51.4 \%$ & $54.0 \%$ & $57.7 \%$ & $55.6 \%$ & $52.0 \%$ & $50.8 \%$ \\
\hline & :paired t-test & 1.719 & 0.973 & 1.450 & 1.376 & $1.996^{*}$ & 0.951 & -0.086 & -0.096 \\
\hline & :Wilcoxon test & $1.825^{*}$ & 1.534 & $2.043^{*}$ & $2.218^{*}$ & $2.802 * *$ & $2.133 *$ & 0.515 & -0.112 \\
\hline \multirow[t]{9}{*}{ FRANCE } & Mean Ret.(\%) - Active Strategy & 0.519 & 0.435 & 0.484 & 0.483 & 0.562 & 0.266 & -0.024 & 0.003 \\
\hline & Mean Ret.(\%) - Passive Equal Weighted Portfolio & 0.006 & -0.015 & -0.018 & -0.040 & -0.042 & -0.042 & -0.050 & -0.045 \\
\hline & Excess Return (\%) & $0.513^{*}$ & 0.450 & 0.502 & 0.523 & 0.604 & 0.308 & 0.025 & 0.048 \\
\hline & Std. Dev.(\%) - Active Strategy & 3.226 & 3.424 & 3.488 & 3.568 & 3.535 & 3.582 & 3.693 & 3.518 \\
\hline & Std. Dev.(\%) - Passive Equal Weighted Portfolio & 1.751 & 1.725 & 1.729 & 1.724 & 1.734 & 1.705 & 1.716 & 1.724 \\
\hline & Infor. Ratio & 0.145 & 0.113 & 0.127 & 0.128 & 0.152 & 0.076 & 0.006 & 0.012 \\
\hline & Prob > Passive Equal Weighted Portfolio (\%) & $56.5 \%$ & $53.5 \%$ & $54.2 \%$ & $57.8 \%$ & $57.7 \%$ & $57.6 \%$ & $53.0 \%$ & $49.2 \%$ \\
\hline & :paired t-test & $2.125^{*}$ & 1.664 & 1.864 & 1.858 & $2.198^{*}$ & 1.085 & 0.088 & 0.171 \\
\hline & :Wilcoxon test & $2.472 * *$ & $2.022^{*}$ & $2.346^{* *}$ & $3.187 * *$ & $3.185^{* *}$ & $2.303^{*}$ & 0.834 & 0.289 \\
\hline \multirow[t]{9}{*}{ ITALY } & Mean Ret.(\%) - Active Strategy & 0.326 & 0.475 & 0.452 & 0.528 & 0.522 & 0.377 & 0.095 & 0.080 \\
\hline & Mean Ret.(\%) - Passive Equal Weighted Portfolio & -0.124 & -0.147 & -0.145 & -0.172 & -0.171 & -0.177 & -0.175 & -0.172 \\
\hline & Excess Return (\%) & 0.451 & 0.622 & 0.597 & 0.699 & 0.693 & 0.554 & 0.270 & 0.252 \\
\hline & Std. Dev.(\%) - Active Strategy & 3.292 & 3.525 & 3.453 & 3.464 & 3.536 & 3.642 & 3.657 & 3.615 \\
\hline & Std. Dev.(\%) - Passive Equal Weighted Portfolio & 1.960 & 1.937 & 1.941 & 1.938 & 1.952 & 1.950 & 1.960 & 1.971 \\
\hline & Infor. Ratio & 0.122 & 0.154 & 0.149 & 0.180 & 0.179 & 0.135 & 0.066 & 0.063 \\
\hline & Prob > Passive Equal Weighted Portfolio (\%) & $54.6 \%$ & $57.7 \%$ & $56.1 \%$ & $58.8 \%$ & $59.1 \%$ & $61.0 \%$ & $57.9 \%$ & $55.3 \%$ \\
\hline & :paired t-test & 1.790 & $2.265^{*}$ & $2.184^{*}$ & $2.615^{* *}$ & $2.587^{* *}$ & 1.940 & 0.932 & 0.887 \\
\hline & :Wilcoxon test & $2.190 *$ & $2.972 * *$ & $3.099 * *$ & $3.845^{* *}$ & $3.709 * *$ & $3.772 * *$ & $2.266^{*}$ & 1.514 \\
\hline
\end{tabular}

Table 5 presents the results of Strategy One back tested over various formation look back periods (from 1 to 18 months) utlising raw currency returns as the source of momentum. Excess return refers to profits generated by the strategy which are greater than the passive buy-and-hold equal weighted portfolio of the respective six foreign currencies. The parametric paired t-test and the non-parametric Wilcoxon test is shown to test the statistical significance of the excess returns relative to the passive equal weighted long only portfolio,

** and * indicate statistical significance at the $1 \%$ and $5 \%$ levels, respectively. 
TABLE 5

Strategy One Results- 1980 to 1998

\section{Panel B: Source of Momentum- Interest Adjusted Returns}

\begin{tabular}{|c|c|c|c|c|c|c|c|c|c|}
\hline \multicolumn{2}{|c|}{ Momentum Formation Period (in months): } & \multirow{2}{*}{$\begin{array}{c}1 \\
0.728\end{array}$} & \multirow{2}{*}{$\begin{array}{c}2 \\
0.593\end{array}$} & \multirow{2}{*}{$\begin{array}{c}3 \\
0.647\end{array}$} & \multirow{2}{*}{$\begin{array}{c}\mathbf{6} \\
0.505\end{array}$} & \multirow{2}{*}{$\begin{array}{c}9 \\
0.298\end{array}$} & \multirow{2}{*}{$\begin{array}{c}12 \\
0.388\end{array}$} & \multirow{2}{*}{$\begin{array}{c}15 \\
0.153\end{array}$} & \multirow{2}{*}{$\begin{array}{c}18 \\
0.142\end{array}$} \\
\hline CANADA & Mean Ret.(\%) - Active Strategy & & & & & & & & \\
\hline & Mean Ret.(\%) - Passive Equal Weighted Portfolio & 0.055 & 0.077 & 0.086 & 0.127 & 0.160 & 0.152 & 0.168 & 0.171 \\
\hline & Excess Return (\%) & 0.673 & 0.516 & 0.561 & 0.378 & 0.138 & 0.236 & -0.015 & -0.030 \\
\hline & Std. Dev.(\%) - Active Strategy & 3.340 & 3.563 & 3.526 & 3.765 & 3.899 & 3.990 & 3.927 & 3.921 \\
\hline & Std. Dev.(\%) - Passive Equal Weighted Portfolio & 2.561 & 2.547 & 2.550 & 2.543 & 2.543 & 2.559 & 2.572 & 2.580 \\
\hline & Infor. Ratio & 0.156 & 0.119 & 0.130 & 0.083 & 0.030 & 0.049 & -0.003 & -0.006 \\
\hline & Prob > Passive Equal Weighted Portfolio (\%) & $58.33 \%$ & $55.35 \%$ & $58.41 \%$ & $58.29 \%$ & $61.06 \%$ & $61.95 \%$ & $58.91 \%$ & $55.78 \%$ \\
\hline & : paired t-test & $2.290 *$ & 1.745 & 1.897 & 1.208 & 0.427 & 0.698 & -0.044 & -0.089 \\
\hline & : Wilcoxon test & $2.854 * *$ & $2.257 *$ & $2.678^{*}$ & $2.192^{*}$ & 1.637 & $2.105^{*}$ & 1.075 & 0.775 \\
\hline \multirow[t]{9}{*}{ U.K. } & Mean Ret.(\%) - Active Strategy & 0.514 & 0.549 & 0.699 & 0.505 & 0.409 & 0.572 & 0.244 & 0.044 \\
\hline & Mean Ret.(\%) - Passive Equal Weighted Portfolio & 0.055 & 0.068 & 0.039 & 0.039 & -0.008 & 0.002 & -0.002 & -0.007 \\
\hline & Excess Return (\%) & 0.459 & 0.481 & $0.660^{*}$ & 0.466 & 0.416 & 0.570 & 0.246 & 0.051 \\
\hline & Std. Dev.(\%) - Active Strategy & 3.240 & 3.586 & 3.563 & 3.659 & 3.861 & 3.882 & 3.781 & 3.961 \\
\hline & Std. Dev.(\%) - Passive Equal Weighted Portfolio & 2.368 & 2.366 & 2.334 & 2.345 & 2.315 & 2.296 & 2.311 & 2.325 \\
\hline & Infor. Ratio & 0.120 & 0.113 & 0.154 & 0.104 & 0.087 & 0.120 & 0.052 & 0.010 \\
\hline & Prob > Passive Equal Weighted Portfolio (\%) & $53.70 \%$ & $53.49 \%$ & $55.61 \%$ & $55.92 \%$ & $56.73 \%$ & $59.02 \%$ & $54.46 \%$ & $52.26 \%$ \\
\hline & : paired t-test & 1.763 & 1.650 & $2.250^{*}$ & 1.508 & 1.259 & 1.722 & 0.741 & 0.147 \\
\hline & : Wilcoxon test & $2.316^{*}$ & $2.216^{*}$ & $3.062 * *$ & $2.220 *$ & $2.568 * *$ & $3.546 * *$ & $2.037 *$ & 1.196 \\
\hline \multirow[t]{9}{*}{ U.S.A. } & Mean Ret.(\%) - Active Strategy & 0.499 & 0.432 & 0.646 & 0.622 & 0.277 & 0.334 & 0.132 & 0.104 \\
\hline & Mean Ret.(\%) - Passive Equal Weighted Portfolio & 0.122 & 0.144 & 0.155 & 0.193 & 0.219 & 0.192 & 0.230 & 0.237 \\
\hline & Excess Return (\%) & 0.377 & 0.288 & 0.491 & 0.429 & 0.058 & 0.143 & -0.098 & -0.133 \\
\hline & Std. Dev.(\%) - Active Strategy & 3.330 & 3.462 & 3.611 & 3.445 & 3.820 & 3.876 & 3.777 & 3.843 \\
\hline & Std. Dev.(\%) - Passive Equal Weighted Portfolio & 2.501 & 2.486 & 2.487 & 2.474 & 2.468 & 2.473 & 2.468 & 2.473 \\
\hline & Infor. Ratio & 0.085 & 0.067 & 0.109 & 0.102 & 0.013 & 0.030 & -0.021 & -0.028 \\
\hline & Prob > Passive Equal Weighted Portfolio (\%) & $51.85 \%$ & $46.05 \%$ & $55.14 \%$ & $53.08 \%$ & $56.25 \%$ & $57.56 \%$ & $53.47 \%$ & $52.76 \%$ \\
\hline & : paired t-test & 1.245 & 0.989 & 1.600 & 1.484 & 0.181 & 0.430 & -0.298 & -0.397 \\
\hline & : Wilcoxon test & $1.814^{*}$ & 1.390 & $2.021 *$ & $2.228^{*}$ & 1.366 & $1.782^{*}$ & 0.737 & 0.425 \\
\hline \multirow[t]{9}{*}{ JAPAN } & Mean Ret.(\%) - Active Strategy & 0.544 & 0.555 & 0.554 & 0.438 & 0.426 & 0.388 & 0.180 & 0.148 \\
\hline & Mean Ret.(\%) - Passive Equal Weighted Portfolio & 0.092 & 0.101 & 0.100 & 0.090 & 0.085 & 0.088 & 0.056 & 0.035 \\
\hline & Excess Return (\%) & 0.452 & 0.454 & 0.454 & 0.348 & 0.340 & 0.300 & 0.123 & 0.113 \\
\hline & Std. Dev.(\%) - Active Strategy & 3.021 & 3.257 & 3.322 & 3.405 & 3.465 & 3.323 & 3.347 & 3.388 \\
\hline & Std. Dev.(\%) - Passive Equal Weighted Portfolio & 2.963 & 2.967 & 2.974 & 2.993 & 2.996 & 2.978 & 2.986 & 2.981 \\
\hline & Infor. Ratio & 0.101 & 0.100 & 0.099 & 0.075 & 0.072 & 0.066 & 0.027 & 0.025 \\
\hline & Prob > Passive Equal Weighted Portfolio (\%) & $49.07 \%$ & $48.37 \%$ & $50.47 \%$ & $51.18 \%$ & $50.00 \%$ & $51.22 \%$ & $50.99 \%$ & $50.75 \%$ \\
\hline & : paired t-test & 1.486 & 1.473 & 1.444 & 1.095 & 1.034 & 0.944 & 0.388 & 0.375 \\
\hline & : Wilcoxon test & 1.168 & 1.30 & 1.496 & 1.505 & 1.474 & 1.376 & 0.675 & 0.549 \\
\hline
\end{tabular}




\begin{tabular}{|c|c|c|c|c|c|c|c|c|c|}
\hline \multirow[t]{9}{*}{ GERMANY } & Mean Ret.(\%) - Active Strategy & 0.558 & 0.600 & 0.726 & 0.745 & 0.341 & 0.421 & 0.172 & 0.060 \\
\hline & Mean Ret.(\%) - Passive Equal Weighted Portfolio & 0.112 & 0.089 & 0.098 & 0.080 & 0.073 & 0.098 & 0.089 & 0.099 \\
\hline & Excess Return (\%) & 0.446 & 0.511 & $0.628 *$ & $0.665^{*}$ & 0.268 & 0.323 & 0.083 & -0.039 \\
\hline & Std. Dev.(\%) - Active Strategy & 3.317 & 3.580 & 3.535 & 3.546 & 3.872 & 3.839 & 3.834 & 4.034 \\
\hline & Std. Dev.(\%) - Passive Equal Weighted Portfolio & 1.818 & 1.790 & 1.789 & 1.787 & 1.799 & 1.775 & 1.784 & 1.794 \\
\hline & Infor. Ratio & 0.124 & 0.125 & 0.160 & 0.165 & 0.061 & 0.074 & 0.019 & -0.008 \\
\hline & Prob > Passive Equal Weighted Portfolio (\%) & $50.93 \%$ & $52.56 \%$ & $56.07 \%$ & $57.35 \%$ & $58.65 \%$ & $59.02 \%$ & $57.43 \%$ & $53.77 \%$ \\
\hline & : paired t-test & 1.828 & 1.837 & $2.340 *$ & $2.398 *$ & 0.877 & 1.059 & 0.267 & -0.118 \\
\hline & : Wilcoxon test & $2.258 *$ & $2.495 * *$ & $2.959 * *$ & $3.444 * *$ & $2.581 * *$ & $3.007 * *$ & $1.796 *$ & 1.138 \\
\hline \multirow[t]{9}{*}{ FRANCE } & Mean Ret.(\%) - Active Strategy & 0.677 & 0.549 & 0.726 & 0.685 & 0.455 & 0.411 & 0.126 & -0.034 \\
\hline & Mean Ret.(\%) - Passive Equal Weighted Portfolio & 0.006 & -0.015 & -0.018 & -0.040 & -0.042 & -0.042 & -0.050 & -0.045 \\
\hline & Excess Return (\%) & $0.671^{* *}$ & $0.564 *$ & $0.744 * *$ & $0.725^{* *}$ & 0.497 & 0.452 & 0.176 & 0.011 \\
\hline & Std. Dev.(\%) - Active Strategy & 3.279 & 3.599 & 3.551 & 3.641 & 3.925 & 3.929 & 3.907 & 3.949 \\
\hline & Std. Dev.(\%) - Passive Equal Weighted Portfolio & 1.751 & 1.725 & 1.729 & 1.724 & 1.734 & 1.705 & 1.716 & 1.724 \\
\hline & Infor. Ratio & 0.183 & 0.137 & 0.190 & 0.180 & 0.116 & 0.105 & 0.040 & 0.002 \\
\hline & Prob > Passive Equal Weighted Portfolio (\%) & $58.80 \%$ & $54.88 \%$ & $61.68 \%$ & $63.51 \%$ & $61.54 \%$ & $61.95 \%$ & $56.93 \%$ & $53.77 \%$ \\
\hline & : paired t-test & $2.683^{* *}$ & $2.011^{*}$ & $2.784 * *$ & $2.613^{* *}$ & 1.666 & 1.509 & 0.575 & 0.034 \\
\hline & : Wilcoxon test & $3.518 * *$ & $2.766^{* *}$ & $3.631 * *$ & $4.182 * *$ & $3.341^{* *}$ & $3.470 * *$ & $1.960 *$ & 1.374 \\
\hline \multirow[t]{9}{*}{ ITALY } & Mean Ret.(\%) - Active Strategy & 0.508 & 0.609 & 0.775 & 0.735 & 0.448 & 0.509 & 0.248 & 0.194 \\
\hline & Mean Ret.(\%) - Passive Equal Weighted Portfolio & -0.124 & -0.147 & -0.145 & -0.172 & -0.171 & -0.177 & -0.175 & -0.172 \\
\hline & Excess Return (\%) & 0.632 & 0.756 & 0.920 & 0.907 & 0.619 & 0.686 & 0.423 & 0.366 \\
\hline & Std. Dev.(\%) - Active Strategy & 3.468 & 3.637 & 3.446 & 3.482 & 3.858 & 3.852 & 3.852 & 3.887 \\
\hline & Std. Dev.(\%) - Passive Equal Weighted Portfolio & 1.960 & 1.937 & 1.941 & 1.938 & 1.952 & 1.950 & 1.960 & 1.971 \\
\hline & Infor. Ratio & 0.163 & 0.181 & 0.228 & 0.230 & 0.142 & 0.159 & 0.098 & 0.081 \\
\hline & Prob > Passive Equal Weighted Portfolio (\%) & $56.94 \%$ & $57.67 \%$ & $61.21 \%$ & $61.61 \%$ & $62.50 \%$ & $65.85 \%$ & $61.88 \%$ & $59.80 \%$ \\
\hline & : paired t-test & $2.399 *$ & $2.648^{* *}$ & $3.339 * *$ & $3.337 * *$ & $2.049 *$ & $2.272 *$ & 1.387 & 1.142 \\
\hline & : Wilcoxon test & $3.162 * *$ & $3.467 * *$ & $4.314^{* *}$ & $4.620 * *$ & $3.996 * *$ & $4.388^{* *}$ & $2.953 * *$ & $2.703^{* *}$ \\
\hline
\end{tabular}

Table 5 presents the results of Strategy One back tested over various formation look back periods (from 1 to 18 months) utlising interest adjusted returns as the source of momentum. Excess return refers to profits generated by the strategy which are greater than the passive buy-and-hold equal weighted portfolio of the respective six foreign currencies. The parametric paired t-test and the non-parametric Wilcoxon test is shown to test the statistical significance of the excess returns relative to the passive equal weighted long only portfolio, ** and * indicate statistical significance at the $1 \%$ and $5 \%$ levels, respectively. 
TABLE 6

Strategy Two Results - 1980 to 1998

\section{Panel A: Source of Momentum - Raw Currency Returns}

Momentum Formation Period (in months):

CANADA Mean Ret.(\%) - Active Strategy

Mean Ret.(\%) - Passive Equal Weighted Portfolio

Excess Return (\%)

Std. Dev.(\%) - Active Strategy

Std. Dev.(\%) - Passive Equal Weighted Portfolio

Infor. Ratio

Prob > Passive Equal Weighted Portfolio (\%)

: paired t-test

: Wilcoxon test
Mean Ret.(\%) - Active Strategy

Mean Ret.(\%) - Passive Equal Weighted Portfolio

Excess Return (\%)

Std. Dev.(\%) - Active Strategy

Std. Dev.(\%) - Passive Equal Weighted Portfolio

Infor. Ratio

Prob > Passive Equal Weighted Portfolio (\%)

: paired t-test

: Wilcoxon test
1

2

3

6

9

12

15 18

\begin{tabular}{cccccccc}
0.232 & 0.271 & 0.234 & 0.281 & 0.394 & 0.240 & -0.012 & -0.004 \\
0.055 & 0.077 & 0.086 & 0.127 & 0.160 & 0.152 & 0.168 & 0.171 \\
0.177 & 0.194 & 0.148 & 0.154 & 0.234 & 0.088 & -0.180 & -0.176 \\
2.456 & 2.484 & 2.602 & 2.648 & 2.601 & 2.475 & 2.588 & 2.482 \\
2.561 & 2.547 & 2.550 & 2.543 & 2.543 & 2.559 & 2.572 & 2.580 \\
0.048 & 0.052 & 0.039 & 0.043 & 0.061 & 0.024 & -0.048 & -0.049 \\
$53.70 \%$ & $52.56 \%$ & $56.07 \%$ & $54.50 \%$ & $56.73 \%$ & $55.61 \%$ & $50.99 \%$ & $50.25 \%$ \\
0.706 & 0.768 & 0.575 & 0.617 & 0.887 & 0.341 & -0.681 & -0.696 \\
0.799 & 1.045 & 0.936 & 0.870 & 1.347 & 0.905 & -0.271 & -0.402 \\
\hline
\end{tabular}

\begin{tabular}{cccccccc}
0.274 & 0.394 & 0.445 & 0.256 & 0.471 & 0.327 & 0.147 & 0.011 \\
0.055 & 0.068 & 0.039 & 0.039 & -0.008 & 0.002 & -0.002 & -0.007 \\
0.219 & 0.326 & 0.406 & 0.217 & 0.479 & 0.325 & 0.149 & 0.018 \\
2.829 & 2.790 & 2.909 & 2.984 & 3.028 & 2.846 & 2.894 & 2.864 \\
2.368 & 2.366 & 2.334 & 2.345 & 2.315 & 2.296 & 2.311 & 2.325 \\
0.060 & 0.089 & 0.109 & 0.057 & 0.122 & 0.087 & 0.039 & 0.005 \\
$52.31 \%$ & $57.21 \%$ & $56.07 \%$ & $53.55 \%$ & $57.69 \%$ & $54.15 \%$ & $51.98 \%$ & $52.26 \%$ \\
0.877 & 1.309 & 1.589 & 0.830 & 1.762 & 1.250 & 0.553 & 0.065 \\
1.548 & 1.534 & $1.998^{*}$ & 1.452 & $2.701^{* *}$ & $2.313^{*}$ & 1.485 & 0.711 \\
\hline
\end{tabular}

U.S.A. $\quad$ Mean Ret.(\%) - Active Strategy

\begin{tabular}{cccccccc}
0.156 & 0.185 & 0.138 & 0.283 & 0.315 & 0.201 & -0.022 & -0.046 \\
0.122 & 0.144 & 0.155 & 0.193 & 0.219 & 0.192 & 0.230 & 0.237 \\
0.034 & 0.041 & -0.017 & 0.091 & 0.096 & 0.009 & -0.252 & -0.283 \\
2.529 & 2.455 & 2.618 & 2.629 & 2.598 & 2.466 & 2.469 & 2.463 \\
2.501 & 2.486 & 2.487 & 2.474 & 2.468 & 2.473 & 2.468 & 2.473 \\
0.009 & 0.011 & -0.005 & 0.026 & 0.026 & 0.002 & -0.071 & -0.079 \\
$50.46 \%$ & $50.23 \%$ & $51.40 \%$ & $53.55 \%$ & $51.44 \%$ & $51.22 \%$ & $47.52 \%$ & $46.73 \%$ \\
0.134 & 0.168 & -0.066 & 0.376 & 0.379 & 0.035 & -1.011 & -1.117 \\
0.193 & 0.35100 & 0.057 & 0.676 & 0.78100 & 0.653 & -0.588 & -0.852 \\
\hline
\end{tabular}

Mean Ret.(\%) - Passive Equal Weighted Portfolio

Excess Return (\%)

Std. Dev.(\%) - Active Strategy

Std. Dev.(\%) - Passive Equal Weighted Portfolio Infor. Ratio

Prob > Passive Equal Weighted Portfolio (\%)

: paired t-test

: Wilcoxon test

JAPAN Mean Ret.(\%) - Active Strategy

Mean Ret.(\%) - Passive Equal Weighted Portfolio

Excess Return (\%)

Std. Dev.(\%) - Active Strategy

Std. Dev.(\%) - Passive Equal Weighted Portfolio

Infor. Ratio

Prob > Passive Equal Weighted Portfolio (\%)

: paired t-test

: Wilcoxon test

\begin{tabular}{cccccccc}
0.381 & 0.379 & 0.342 & 0.325 & 0.400 & 0.269 & 0.073 & 0.007 \\
0.092 & 0.101 & 0.100 & 0.090 & 0.085 & 0.088 & 0.056 & 0.035 \\
0.288 & 0.278 & 0.241 & 0.236 & 0.315 & 0.181 & 0.017 & -0.029 \\
2.677 & 2.807 & 2.878 & 2.866 & 2.945 & 2.869 & 2.883 & 2.872 \\
2.963 & 2.967 & 2.974 & 2.993 & 2.996 & 2.978 & 2.986 & 2.981 \\
0.069 & 0.066 & 0.056 & 0.054 & 0.072 & 0.042 & 0.004 & -0.007 \\
$50.46 \%$ & $51.63 \%$ & $50.47 \%$ & $48.34 \%$ & $49.52 \%$ & $50.73 \%$ & $49.50 \%$ & $49.25 \%$ \\
1.008 & 0.962 & 0.818 & 0.785 & 1.036 & 0.601 & 0.056 & -0.093 \\
0.485 & 0.50900 & 0.483 & 0.625 & 1.12200 & 0.651 & -0.050 & -0.267 \\
\hline
\end{tabular}




\begin{tabular}{|c|c|c|c|c|c|c|c|c|c|}
\hline \multirow[t]{9}{*}{ GERMANY } & Mean Ret.(\%) - Active Strategy & 0.251 & 0.341 & 0.425 & 0.294 & 0.438 & 0.319 & 0.078 & 0.024 \\
\hline & Mean Ret.(\%) - Passive Equal Weighted Portfolio & 0.112 & 0.089 & 0.098 & 0.080 & 0.073 & 0.098 & 0.089 & 0.099 \\
\hline & Excess Return (\%) & 0.139 & 0.252 & 0.327 & 0.214 & 0.365 & 0.220 & -0.011 & -0.074 \\
\hline & Std. Dev.(\%) - Active Strategy & 2.768 & 2.785 & 2.847 & 2.869 & 2.992 & 2.862 & 2.877 & 2.863 \\
\hline & Std. Dev.(\%) - Passive Equal Weighted Portfolio & 1.818 & 1.790 & 1.789 & 1.787 & 1.799 & 1.775 & 1.784 & 1.794 \\
\hline & Infor. Ratio & 0.043 & 0.076 & 0.098 & 0.059 & 0.102 & 0.065 & -0.003 & -0.021 \\
\hline & Prob > Passive Equal Weighted Portfolio (\%) & $51.39 \%$ & $53.95 \%$ & $52.34 \%$ & $53.08 \%$ & $53.85 \%$ & $54.15 \%$ & $50.00 \%$ & $49.25 \%$ \\
\hline & :paired t-test & 0.627 & 1.119 & 1.428 & 0.861 & 1.475 & 0.926 & -0.045 & -0.294 \\
\hline & :Wilcoxon test & 0.950 & 1.227 & 1.547 & 1.535 & $2.188^{*}$ & $1.888^{*}$ & 0.601 & 0.039 \\
\hline \multirow[t]{9}{*}{ FRANCE } & Mean Ret.(\%) - Active Strategy & 0.283 & 0.355 & 0.443 & 0.201 & 0.420 & 0.316 & 0.098 & -0.080 \\
\hline & Mean Ret.(\%) - Passive Equal Weighted Portfolio & 0.006 & -0.015 & -0.018 & -0.040 & -0.042 & -0.042 & -0.050 & -0.045 \\
\hline & Excess Return (\%) & 0.277 & 0.371 & $0.461^{*}$ & 0.241 & 0.462 & 0.357 & 0.147 & -0.035 \\
\hline & Std. Dev.(\%) - Active Strategy & 2.859 & 2.742 & 2.921 & 2.401 & 2.985 & 2.913 & 2.973 & 2.893 \\
\hline & Std. Dev.(\%) - Passive Equal Weighted Portfolio & 1.751 & 1.725 & 1.729 & 1.724 & 1.734 & 1.705 & 1.716 & 1.724 \\
\hline & Infor. Ratio & 0.085 & 0.113 & 0.136 & 0.093 & 0.133 & 0.107 & 0.043 & -0.010 \\
\hline & Prob > Passive Equal Weighted Portfolio (\%) & $50.93 \%$ & $50.23 \%$ & $51.40 \%$ & $53.08 \%$ & $58.17 \%$ & $58.54 \%$ & $55.45 \%$ & $53.27 \%$ \\
\hline & :paired t-test & 1.242 & 1.657 & $1.996^{*}$ & 1.356 & 1.920 & 1.528 & 0.611 & -0.140 \\
\hline & :Wilcoxon test & $1.695 *$ & $1.975^{*}$ & $2.249 *$ & $1.872^{*}$ & $2.774^{* *}$ & $2.795^{* *}$ & 1.452 & 0.543 \\
\hline \multirow[t]{9}{*}{ ITALY } & Mean Ret.(\%) - Active Strategy & 0.304 & 0.340 & 0.472 & 0.329 & 0.490 & 0.344 & 0.163 & -0.016 \\
\hline & Mean Ret.(\%) - Passive Equal Weighted Portfolio & -0.124 & -0.147 & -0.145 & -0.172 & -0.171 & -0.177 & -0.175 & -0.172 \\
\hline & Excess Return (\%) & 0.428 & 0.487 & 0.617 & 0.501 & 0.661 & 0.521 & 0.338 & 0.156 \\
\hline & Std. Dev.(\%) - Active Strategy & 2.828 & 2.830 & 2.949 & 2.993 & 2.992 & 2.957 & 3.042 & 2.952 \\
\hline & Std. Dev.(\%) - Passive Equal Weighted Portfolio & 1.960 & 1.937 & 1.941 & 1.938 & 1.952 & 1.950 & 1.960 & 1.971 \\
\hline & Infor. Ratio & 0.126 & 0.147 & 0.181 & 0.141 & 0.189 & 0.147 & 0.094 & 0.044 \\
\hline & Prob > Passive Equal Weighted Portfolio (\%) & $56.02 \%$ & $57.21 \%$ & $57.01 \%$ & $60.19 \%$ & $64.90 \%$ & $65.85 \%$ & $60.40 \%$ & $59.80 \%$ \\
\hline & :paired t-test & 1.854 & $2.158^{*}$ & $2.642^{* *}$ & $2.042 *$ & $2.726^{* *}$ & $2.106^{*}$ & 1.331 & 0.627 \\
\hline & :Wilcoxon test & $2.577^{* *}$ & $2.549 * *$ & $3.172^{* *}$ & $3.336 * *$ & $4.035 * *$ & $4.182 * *$ & $2.879 * *$ & $1.970 *$ \\
\hline
\end{tabular}

Table 6 presents the results of Strategy Two back tested over various formation look back periods (from 1 to 18 months) utlising raw currency returns as the source of momentum. Excess return refers to profits generated by the strategy which are greater than the passive buy-and-hold equal weighted portfolio of the respective six foreign currencies. The parametric paired t-test and the non-parametric Wilcoxon test is shown to test the statistical significance of the excess returns relative to the passive equal weighted long only portfolio, ** and * indicate statistical significance at the $1 \%$ and $5 \%$ levels, respectively. 
TABLE 6

Strategy Two Results - 1980 to 1998

Panel B: Source of Momentum- Interest Adjusted Returns

Momentum Formation Period (in months):

1

$2 \quad 3 \quad 6$

9

12

$15 \quad 18$

CANADA Mean Ret.(\%) - Active Strategy

Mean Ret.(\%) - Passive Equal Weighted Portfolio

Excess Return (\%)

Std. Dev.(\%) - Active Strategy

Std. Dev.(\%) - Passive Equal Weighted Portfolio

Infor. Ratio

Prob > Passive Equal Weighted Portfolio (\%)

: paired t-test

: Wilcoxon test

$\begin{array}{cccccccc}0.313 & 0.438 & 0.330 & 0.338 & 0.422 & 0.259 & 0.172 & 0.067 \\ 0.055 & 0.077 & 0.086 & 0.127 & 0.160 & 0.152 & 0.168 & 0.171 \\ 0.258 & 0.361 & 0.244 & 0.212 & 0.262 & 0.107 & 0.004 & -0.104 \\ 2.439 & 2.430 & 2.571 & 2.724 & 2.702 & 2.570 & 2.525 & 2.487 \\ 2.561 & 2.547 & 2.550 & 2.543 & 2.543 & 2.559 & 2.572 & 2.580 \\ 0.071 & 0.101 & 0.066 & 0.056 & 0.066 & 0.028 & 0.001 & -0.029 \\ 55.09 \% & 56.28 \% & 60.28 \% & 57.82 \% & 62.50 \% & 58.54 \% & 55.94 \% & 51.76 \% \\ 1.045 & 1.476 & 0.970 & 0.815 & 0.957 & 0.396 & 0.016 & -0.414 \\ 1.297 & 1.950 * & 1.527 & 1.357 & 1.707 * & 1.323 & 0.637 & 0.156\end{array}$

U.K. $\quad$ Mean Ret.(\%) - Active Strategy

Mean Ret.(\%) - Passive Equal Weighted Portfolio

Excess Return (\%)

Std. Dev.(\%) - Active Strategy

Std. Dev.(\%) - Passive Equal Weighted Portfolio

Infor. Ratio

Prob > Passive Equal Weighted Portfolio (\%)

: paired t-test

: Wilcoxon test

\begin{tabular}{cccccccc}
0.349 & 0.525 & 0.549 & 0.324 & 0.407 & 0.453 & 0.322 & 0.157 \\
0.055 & 0.068 & 0.039 & 0.039 & -0.008 & 0.002 & -0.002 & -0.007 \\
0.293 & 0.457 & $0.510 *$ & 0.286 & 0.415 & 0.451 & 0.324 & 0.164 \\
2.881 & 2.731 & 2.791 & 3.014 & 3.069 & 3.008 & 2.959 & 2.906 \\
2.368 & 2.366 & 2.334 & 2.345 & 2.315 & 2.296 & 2.311 & 2.325 \\
0.077 & 0.127 & 0.141 & 0.074 & 0.102 & 0.113 & 0.081 & 0.041 \\
$54.63 \%$ & $60.00 \%$ & $58.88 \%$ & $55.92 \%$ & $58.65 \%$ & $59.02 \%$ & $55.94 \%$ & $54.77 \%$ \\
1.126 & 1.856 & $2.062 *$ & 1.073 & 1.471 & 1.621 & 1.156 & 0.578 \\
$1.983 *$ & $2.284 *$ & $2.707 * *$ & $1.910 *$ & $2.731 * *$ & $3.109 * *$ & $2.494 * *$ & 1.512 \\
\hline
\end{tabular}

U.S.A. $\quad$ Mean Ret.(\%) - Active Strategy

Mean Ret.(\%) - Passive Equal Weighted Portfolio

Excess Return (\%)

Std. Dev.(\%) - Active Strategy

Std. Dev.(\%) - Passive Equal Weighted Portfolio

Infor. Ratio

Prob > Passive Equal Weighted Portfolio (\%)

: paired t-test

: Wilcoxon test

\begin{tabular}{cccccccc}
0.204 & 0.422 & 0.298 & 0.359 & 0.384 & 0.221 & 0.051 & 0.003 \\
0.122 & 0.144 & 0.155 & 0.193 & 0.219 & 0.192 & 0.230 & 0.237 \\
0.082 & 0.278 & 0.143 & 0.166 & 0.165 & 0.029 & -0.179 & -0.234 \\
2.538 & 2.402 & 2.598 & 2.605 & 2.656 & 2.589 & 2.551 & 2.431 \\
2.501 & 2.486 & 2.487 & 2.474 & 2.468 & 2.473 & 2.468 & 2.473 \\
0.022 & 0.078 & 0.037 & 0.045 & 0.043 & 0.008 & -0.049 & -0.065 \\
$50.46 \%$ & $55.35 \%$ & $55.61 \%$ & $57.82 \%$ & $56.25 \%$ & $53.66 \%$ & $50.50 \%$ & $49.25 \%$ \\
0.327 & 1.149 & 0.548 & 0.660 & 0.626 & 0.108 & -0.690 & -0.910 \\
0.476 & 1.41900 & 1.072 & 1.182 & 1.26500 & 0.994 & -0.034 & -0.469 \\
\hline & & & & & & & \\
0.505 & 0.471 & 0.376 & 0.374 & 0.354 & 0.346 & 0.266 & 0.178 \\
0.092 & 0.101 & 0.100 & 0.090 & 0.085 & 0.088 & 0.056 & 0.035 \\
0.413 & 0.370 & 0.276 & 0.284 & 0.269 & 0.259 & 0.209 & 0.142 \\
2.652 & 2.716 & 2.791 & 2.895 & 2.928 & 2.844 & 2.844 & 2.784 \\
2.963 & 2.967 & 2.974 & 2.993 & 2.996 & 2.978 & 2.986 & 2.981 \\
0.101 & 0.088 & 0.065 & 0.066 & 0.062 & 0.061 & 0.050 & 0.034 \\
$52.31 \%$ & $53.02 \%$ & $51.40 \%$ & $50.24 \%$ & $50.96 \%$ & $52.68 \%$ & $50.99 \%$ & $49.75 \%$ \\
1.479 & 1.293 & 0.944 & 0.955 & 0.889 & 0.870 & 0.710 & 0.480 \\
0.974 & 1.09900 & 0.715 & 0.958 & 1.02400 & 1.063 & 0.660 & 0.400 \\
\hline
\end{tabular}


GERMANY Mean Ret.(\%) - Active Strategy

Mean Ret.(\%) - Passive Equal Weighted Portfolio

Excess Return (\%)

Std. Dev.(\%) - Active Strategy

Std. Dev.(\%) - Passive Equal Weighted Portfolio

Infor. Ratio

Prob > Passive Equal Weighted Portfolio (\%)

:paired t-test

:Wilcoxon test

\begin{tabular}{cccccccc}
0.384 & 0.522 & 0.461 & 0.365 & 0.359 & 0.367 & 0.321 & 0.194 \\
0.112 & 0.089 & 0.098 & 0.080 & 0.073 & 0.098 & 0.089 & 0.099 \\
0.271 & 0.433 & 0.363 & 0.285 & 0.287 & 0.268 & 0.232 & 0.096 \\
2.780 & 2.753 & 2.790 & 2.962 & 2.989 & 2.895 & 2.909 & 2.805 \\
1.818 & 1.790 & 1.789 & 1.787 & 1.799 & 1.775 & 1.784 & 1.794 \\
0.083 & 0.130 & 0.105 & 0.077 & 0.079 & 0.075 & 0.064 & 0.027 \\
$53.2 \%$ & $56.7 \%$ & $57.0 \%$ & $57.8 \%$ & $56.7 \%$ & $55.1 \%$ & $56.4 \%$ & $55.3 \%$ \\
1.216 & 1.900 & 1.542 & 1.120 & 1.143 & 1.081 & 0.914 & 0.377 \\
1.558 & $2.414 * *$ & $2.236 *$ & $2.105 *$ & $2.064 *$ & $2.538 * *$ & $2.060 *$ & 1.455 \\
\hline & & & & & & & \\
0.491 & 0.553 & 0.541 & 0.370 & 0.396 & 0.418 & 0.357 & 0.194 \\
0.006 & -0.015 & -0.018 & -0.040 & -0.042 & -0.042 & -0.050 & -0.045 \\
$0.484 *$ & $0.568 *$ & $0.558 *$ & 0.410 & 0.438 & 0.459 & 0.407 & 0.239 \\
2.884 & 2.706 & 2.852 & 2.957 & 3.016 & 2.931 & 2.920 & 2.788 \\
1.751 & 1.725 & 1.729 & 1.724 & 1.734 & 1.705 & 1.716 & 1.724 \\
0.146 & 0.170 & 0.165 & 0.115 & 0.125 & 0.135 & 0.118 & 0.072 \\
$54.6 \%$ & $54.4 \%$ & $55.1 \%$ & $57.3 \%$ & $60.6 \%$ & $63.4 \%$ & $61.4 \%$ & $61.3 \%$ \\
$2.151 *$ & $2.497 *$ & $2.410 *$ & 1.666 & 1.799 & 1.928 & 1.672 & 1.009 \\
$2.609 * *$ & $3.267 * *$ & $3.056 * *$ & $2.891 * *$ & $2.970 * *$ & $3.733 * *$ & $3.110 * *$ & $2.242 *$ \\
\hline
\end{tabular}

\begin{tabular}{cccccccc}
0.413 & 0.492 & 0.520 & 0.375 & 0.474 & 0.407 & 0.288 & 0.187 \\
-0.124 & -0.147 & -0.145 & -0.172 & -0.171 & -0.177 & -0.175 & -0.172 \\
0.537 & 0.638 & 0.665 & 0.547 & 0.644 & 0.584 & 0.463 & 0.359 \\
2.819 & 2.733 & 2.852 & 2.979 & 3.070 & 2.984 & 2.936 & 2.918 \\
1.960 & 1.937 & 1.941 & 1.938 & 1.952 & 1.950 & 1.960 & 1.971 \\
0.158 & 0.192 & 0.194 & 0.153 & 0.181 & 0.163 & 0.130 & 0.102 \\
$57.4 \%$ & $57.2 \%$ & $63.1 \%$ & $61.6 \%$ & $66.3 \%$ & $67.8 \%$ & $63.4 \%$ & $63.3 \%$ \\
$2.322 *$ & $2.813 * *$ & $2.844 * *$ & $2.216 *$ & $2.610 * *$ & $2.330 *$ & 1.847 & 1.444 \\
$3.031 * *$ & $3.719 * *$ & $3.983 * *$ & $3.699 * *$ & $4.479 * *$ & $4.792 * *$ & $3.816 * *$ & $2.865 * *$ \\
\hline
\end{tabular}

Table 6 presents the results of Strategy Two back tested over various formation look back periods (from 1 to 18 months) utlising interest adjusted returns as the source of momentum. Excess return refers to profits generated by the strategy which are greater than the passive buy-and-hold equal weighted portfolio of the respective six foreign currencies. The parametric paired t-test and the non-parametric Wilcoxon test is shown to test the statistical significance of the excess returns relative to the passive equal weighted long only portfolio,

** and * indicate statistical significance at the $1 \%$ and $5 \%$ levels, respectively. 
TABLE 7

Strategy One Results - 1999 to 2004

\section{Panel A: Source of Momentum - Raw Currency Returns}

Momentum Formation Period (in months):

CANADA Mean Ret.(\%) - Active Strategy

Mean Ret.(\%) - Passive Equal Weighted Portfolio

Excess Return (\%)

Std. Dev.(\%) - Active Strategy

Std. Dev.(\%) - Passive Equal Weighted Portfolio Infor. Ratio

Prob > Passive Equal Weighted Portfolio (\%)

: paired t-test

: Wilcoxon test

U.K. $\quad$ Mean Ret.(\%) - Active Strategy

Mean Ret.(\%) - Passive Equal Weighted Portfolio

Excess Return (\%)

Std. Dev.(\%) - Active Strategy

Std. Dev.(\%) - Passive Equal Weighted Portfolio

Infor. Ratio

Prob > Passive Equal Weighted Portfolio (\%)

: paired t-test

: Wilcoxon test

1

2

\begin{tabular}{cccccccc}
0.583 & 0.029 & 0.358 & 0.199 & 1.052 & 0.534 & 0.401 & 0.653 \\
-0.146 & -0.110 & -0.103 & -0.012 & -0.113 & -0.066 & -0.030 & -0.025 \\
0.776 & 0.187 & 0.509 & 0.262 & 1.219 & 0.658 & 0.492 & 0.744 \\
2.570 & 2.727 & 2.770 & 3.185 & 2.943 & 3.194 & 3.146 & 3.447 \\
2.053 & 2.051 & 2.068 & 2.043 & 1.924 & 1.961 & 1.973 & 2.031 \\
0.227 & 0.011 & 0.129 & 0.062 & 0.357 & 0.167 & 0.127 & 0.190 \\
$58.33 \%$ & $50.85 \%$ & $60.34 \%$ & $50.91 \%$ & $59.62 \%$ & $51.02 \%$ & $50.00 \%$ & $58.14 \%$ \\
1.927 & 0.462 & 1.229 & 0.550 & $2.524 *$ & 1.160 & 0.865 & 1.115 \\
$1.963 *$ & 0.326 & 1.231 & 0.517 & $2.022 *$ & 0.963 & 0.664 & 1.360 \\
\hline
\end{tabular}

\begin{tabular}{cccccccc}
0.751 & 0.258 & 0.122 & 0.116 & 0.767 & 0.527 & 0.327 & 0.516 \\
-0.188 & -0.211 & -0.194 & -0.221 & -0.218 & -0.256 & -0.264 & -0.351 \\
0.893 & 0.423 & 0.268 & 0.287 & 0.932 & 0.727 & 0.531 & 0.803 \\
3.136 & 2.914 & 3.143 & 3.100 & 3.016 & 3.186 & 3.130 & 3.364 \\
1.860 & 1.868 & 1.879 & 1.912 & 1.930 & 1.961 & 1.987 & 1.958 \\
0.239 & 0.089 & 0.039 & 0.038 & 0.254 & 0.166 & 0.104 & 0.153 \\
$61.67 \%$ & $54.24 \%$ & $53.45 \%$ & $49.09 \%$ & $57.69 \%$ & $57.14 \%$ & $56.52 \%$ & $67.44 \%$ \\
1.908 & 0.910 & 0.557 & 0.592 & 1.945 & 1.367 & 1.031 & 1.398 \\
$1.727 *$ & 0.686 & 0.569 & 0.858 & $1.749 *$ & 1.346 & 0.867 & 1.455 \\
\hline
\end{tabular}

U.S.A. Mean Ret.(\%) - Active Strategy

Mean Ret.(\%) - Passive Equal Weighted Portfolio

$\begin{array}{lll}0.208 & 0.244 & 0.255\end{array}$

$\begin{array}{lll}-0.077 & -0.448 & -0.345\end{array}$

0.291

0.208

0.231

0.285

0.363

2.777

2.834

2.751

$-0.520$

$-0.001$

$-0.519$

$-0.542$

$-0.307$

Std. Dev.(\%) - Active Strategy

Std. Dev.(\%) - Passive Equal Weighted Portfolio

$\begin{array}{llll}1.815 & 1.808 & 1.822 & 1.860\end{array}$

3.267

3.223

$3.082 \quad 3.319$

$\begin{array}{llllllll}0.046 & -0.073 & -0.034 & -0.067 & 0.062 & -0.090 & -0.085 & 0.016\end{array}$

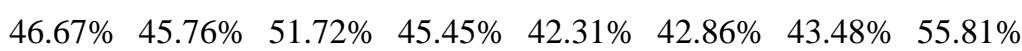

$\begin{array}{llllllll}-0.204 & -1.123 & -0.845 & -1.036 & -0.001 & -0.936 & -1.007 & -0.520\end{array}$

\begin{tabular}{llllllll}
-0.105 & -1.068 & -0.718 & -0.655 & -0.605 & -1.339 & -0.914 & -0.367 \\
\hline
\end{tabular}

: paired t-test

: Wilcoxon test 


\begin{tabular}{|c|c|c|c|c|c|c|c|c|c|}
\hline \multirow[t]{9}{*}{ JAPAN } & Mean Ret.(\%) - Active Strategy & 0.142 & 0.195 & 0.082 & 0.049 & 0.776 & 0.415 & 0.382 & 0.552 \\
\hline & Mean Ret.(\%) - Passive Equal Weighted Portfolio & 0.335 & 0.318 & 0.328 & 0.305 & 0.513 & 0.631 & 0.665 & 0.649 \\
\hline & Excess Return (\%) & -0.217 & -0.147 & -0.270 & -0.282 & 0.236 & -0.245 & -0.313 & -0.131 \\
\hline & Std. Dev.(\%) - Active Strategy & 2.485 & 2.815 & 3.056 & 2.880 & 2.453 & 2.751 & 2.982 & 3.116 \\
\hline & Std. Dev.(\%) - Passive Equal Weighted Portfolio & 2.736 & 2.756 & 2.779 & 2.848 & 2.769 & 2.769 & 2.592 & 2.644 \\
\hline & Infor. Ratio & 0.057 & 0.069 & 0.027 & 0.017 & 0.316 & 0.151 & 0.128 & 0.177 \\
\hline & Prob > Passive Equal Weighted Portfolio (\%) & $53.33 \%$ & $47.46 \%$ & $44.83 \%$ & $49.09 \%$ & $53.85 \%$ & $46.94 \%$ & $50.00 \%$ & $46.51 \%$ \\
\hline & : paired t-test & -0.449 & -0.274 & -0.473 & -0.471 & 0.415 & -0.408 & -0.484 & -0.195 \\
\hline & : Wilcoxon test & -0.588 & -0.315 & -0.657 & -0.176 & 0.709 & -0.416 & -0.476 & -0.600 \\
\hline \multirow[t]{9}{*}{ EURO } & Mean Ret.(\%) - Active Strategy & 0.002 & -0.450 & 0.161 & 0.254 & 0.364 & 0.358 & 0.247 & 0.023 \\
\hline & Mean Ret.(\%) - Passive Equal Weighted Portfolio & 0.002 & -0.031 & -0.076 & -0.152 & -0.191 & -0.349 & -0.481 & -0.470 \\
\hline & Excess Return (\%) & 0.021 & -0.398 & 0.258 & 0.428 & 0.579 & 0.731 & 0.754 & 0.521 \\
\hline & Std. Dev.(\%) - Active Strategy & 2.665 & 2.668 & 2.930 & 3.270 & 3.183 & 3.023 & 2.889 & 3.077 \\
\hline & Std. Dev.(\%) - Passive Equal Weighted Portfolio & 2.399 & 2.406 & 2.402 & 2.434 & 2.461 & 2.422 & 2.403 & 2.402 \\
\hline & Infor. Ratio & 0.001 & -0.169 & 0.055 & 0.078 & 0.114 & 0.118 & 0.085 & 0.007 \\
\hline & Prob > Passive Equal Weighted Portfolio (\%) & $48.33 \%$ & $47.46 \%$ & $53.45 \%$ & $56.36 \%$ & $55.77 \%$ & $53.06 \%$ & $58.70 \%$ & $55.81 \%$ \\
\hline & :paired t-test & 0.041 & -0.806 & 0.496 & 0.783 & 1.013 & 1.320 & 1.348 & 0.931 \\
\hline & :Wilcoxon test & 0.074 & -0.724 & 0.674 & 0.655 & 0.930 & 1.098 & 1.398 & 0.877 \\
\hline
\end{tabular}

Table 7 presents the results of Strategy One back tested over various formation look back periods (from 1 to 18 months) utlising raw currency returns as the source of momentum. Excess return refers to profits generated by the strategy which are greater than the passive buy-and-hold equal weighted portfolio of the respective six foreign currencies. The parametric paired t-test and the non-parametric Wilcoxon test is shown to test the statistical significance of the excess returns relative to the passive equal weighted long only portfolio, ** and $*$ indicate statistical significance at the $1 \%$ and $5 \%$ levels, respectively. 
TABLE 7

Strategy One Results - 1999 to 2004

\section{Panel B: Source of Momentum - Interest Adjusted Returns}

Momentum Formation Period (in months):

1

2

CANADA Mean Ret.(\%) - Active Strategy

Mean Ret.(\%) - Passive Equal Weighted Portfolio

Excess Return (\%)

Std. Dev.(\%) - Active Strategy

Std. Dev.(\%) - Passive Equal Weighted Portfolio Infor. Ratio

Prob $>$ Passive Equal Weighted Portfolio (\%)

: paired t-test

: Wilcoxon test

\begin{tabular}{cccccccc}
0.629 & 0.409 & 0.528 & 0.233 & 1.009 & 0.745 & 0.511 & 0.735 \\
-0.146 & -0.110 & -0.103 & -0.012 & -0.113 & -0.066 & -0.030 & -0.025 \\
0.775 & 0.519 & 0.631 & 0.245 & 1.123 & 0.811 & 0.541 & 0.760 \\
2.979 & 2.950 & 2.773 & 3.129 & 3.197 & 3.274 & 3.142 & 3.430 \\
2.053 & 2.051 & 2.068 & 2.043 & 1.924 & 1.961 & 1.973 & 2.031 \\
0.211 & 0.139 & 0.190 & 0.075 & 0.316 & 0.228 & 0.163 & 0.214 \\
$58.33 \%$ & $57.63 \%$ & $60.34 \%$ & $50.91 \%$ & $55.77 \%$ & $51.02 \%$ & $52.17 \%$ & $51.16 \%$ \\
1.523 & 1.042 & 1.275 & 0.461 & $2.163 *$ & 1.449 & 0.941 & 1.171 \\
$1.653 *$ & 1.085 & 1.568 & 0.828 & $1.931 *$ & 1.375 & 0.773 & 1.619 \\
\hline & & & & & & & \\
0.833 & 0.474 & 0.317 & 0.363 & 0.903 & 0.581 & 0.465 & 0.625 \\
-0.188 & -0.211 & -0.194 & -0.221 & -0.218 & -0.256 & -0.264 & -0.351 \\
1.021 & 0.686 & 0.510 & 0.584 & 1.121 & 0.836 & 0.729 & 0.976 \\
3.233 & 2.942 & 3.212 & 3.134 & 3.011 & 2.919 & 3.160 & 3.381 \\
1.860 & 1.868 & 1.879 & 1.912 & 1.930 & 1.961 & 1.987 & 1.958 \\
0.258 & 0.161 & 0.099 & 0.116 & 0.300 & 0.199 & 0.147 & 0.185 \\
$61.67 \%$ & $57.63 \%$ & $60.34 \%$ & $60.00 \%$ & $61.54 \%$ & $61.22 \%$ & $58.70 \%$ & $60.47 \%$ \\
$2.013 *$ & 1.473 & 0.962 & 1.143 & $2.180 *$ & 1.581 & 1.218 & 1.602 \\
$1.685 *$ & 1.235 & 0.999 & 1.551 & $2.230 *$ & $1.872 *$ & 1.187 & $1.888 *$ \\
\hline & & & & & & & \\
0.377 & 0.132 & 0.087 & 0.211 & 0.207 & -0.049 & 0.178 & 0.342 \\
0.208 & 0.244 & 0.255 & 0.291 & 0.208 & 0.231 & 0.285 & 0.363 \\
0.169 & -0.112 & -0.168 & -0.080 & -0.001 & -0.281 & -0.107 & -0.021 \\
3.208 & 2.772 & 2.962 & 3.013 & 3.442 & 3.040 & 2.960 & 3.534 \\
1.815 & 1.808 & 1.822 & 1.860 & 1.873 & 1.916 & 1.915 & 1.882 \\
0.117 & 0.048 & 0.029 & 0.070 & 0.060 & -0.016 & 0.060 & 0.097 \\
$51.67 \%$ & $45.76 \%$ & $43.10 \%$ & $45.45 \%$ & $44.23 \%$ & $42.86 \%$ & $47.83 \%$ & $48.84 \%$ \\
0.392 & -0.245 & -0.350 & -0.156 & -0.002 & -0.505 & -0.198 & -0.033 \\
0.042 & -0.175 & -0.331 & -0.218 & -0.416 & -0.544 & -0.320 & 0.186 \\
\hline
\end{tabular}




\begin{tabular}{|c|c|c|c|c|c|c|c|c|c|}
\hline \multirow[t]{9}{*}{ JAPAN } & Mean Ret.(\%) - Active Strategy & 0.180 & 0.281 & 0.156 & 0.196 & 0.669 & 0.307 & 0.475 & 0.714 \\
\hline & Mean Ret.(\%) - Passive Equal Weighted Portfolio & 0.335 & 0.318 & 0.328 & 0.305 & 0.513 & 0.631 & 0.665 & 0.649 \\
\hline & Excess Return (\%) & -0.155 & -0.037 & -0.172 & -0.109 & 0.156 & -0.324 & -0.189 & 0.065 \\
\hline & Std. Dev.(\%) - Active Strategy & 2.570 & 2.872 & 3.040 & 2.895 & 2.781 & 2.703 & 3.038 & 3.093 \\
\hline & Std. Dev.(\%) - Passive Equal Weighted Portfolio & 2.736 & 2.756 & 2.779 & 2.848 & 2.769 & 2.769 & 2.592 & 2.644 \\
\hline & Infor. Ratio & 0.070 & 0.098 & 0.051 & 0.068 & 0.241 & 0.114 & 0.156 & 0.231 \\
\hline & Prob > Passive Equal Weighted Portfolio (\%) & $43.33 \%$ & $44.07 \%$ & $41.38 \%$ & $47.27 \%$ & $53.85 \%$ & $38.78 \%$ & $43.48 \%$ & $44.19 \%$ \\
\hline & : paired t-test & -0.328 & -0.068 & -0.324 & -0.196 & 0.266 & -0.585 & -0.321 & 0.098 \\
\hline & : Wilcoxon test & -0.614 & -0.073 & -0.387 & 0.230 & 0.650 & -0.700 & -0.289 & -0.255 \\
\hline \multirow[t]{9}{*}{ EURO } & Mean Ret.(\%) - Active Strategy & 0.136 & -0.131 & 0.408 & 0.308 & 0.481 & 0.557 & 0.405 & 0.436 \\
\hline & Mean Ret.(\%) - Passive Equal Weighted Portfolio & 0.002 & -0.031 & -0.076 & -0.152 & -0.191 & -0.349 & -0.481 & -0.470 \\
\hline & Excess Return (\%) & 0.134 & -0.100 & 0.484 & 0.460 & 0.673 & 0.906 & 0.886 & 0.906 \\
\hline & Std. Dev.(\%) - Active Strategy & 2.517 & 2.777 & 3.217 & 3.344 & 3.022 & 3.007 & 2.944 & 3.149 \\
\hline & Std. Dev.(\%) - Passive Equal Weighted Portfolio & 2.399 & 2.406 & 2.402 & 2.434 & 2.461 & 2.422 & 2.403 & 2.402 \\
\hline & Infor. Ratio & 0.054 & -0.047 & 0.127 & 0.092 & 0.159 & 0.185 & 0.137 & 0.138 \\
\hline & Prob > Passive Equal Weighted Portfolio (\%) & $55.00 \%$ & $50.85 \%$ & $55.17 \%$ & $50.91 \%$ & $57.69 \%$ & $63.27 \%$ & $63.04 \%$ & $62.79 \%$ \\
\hline & :paired t-test & 0.282 & -0.210 & 0.881 & 0.778 & 1.209 & 1.507 & 1.616 & 1.643 \\
\hline & :Wilcoxon test & 0.441 & -0.250 & 1.209 & 0.929 & 1.300 & 1.600 & $1.647 *$ & $1.663 *$ \\
\hline
\end{tabular}

Table 7 presents the results of Strategy One back tested over various formation look back periods (from 1 to 18 months) utlising interest adjusted returns as the source of momentum. Excess return refers to profits generated by the strategy which are greater than the passive buy-and-hold equal weighted portfolio of the respective six foreign currencies. The parametric paired t-test and the non-parametric Wilcoxon test is shown to test the statistical significance of the excess returns relative to the passive equal weighted long only portfolio, ** and * indicate statistical significance at the $1 \%$ and $5 \%$ levels, respectively. 
TABLE 8

Strategy Two Results - 1999 to 2004

Panel A: Source of Momentum - Raw Currency Returns

Momentum Formation Period (in months):

1

2

3

6

9

12

15

18

CANADA Mean Ret.(\%) - Active Strategy

Mean Ret.(\%) - Passive Equal Weighted Portfolio

Excess Return (\%)

Std. Dev.(\%) - Active Strategy

Std. Dev.(\%) - Passive Equal Weighted Portfolio

Infor. Ratio

Prob > Passive Equal Weighted Portfolio (\%)

: paired t-test

: Wilcoxon test

\begin{tabular}{cccccccc}
1.015 & 0.136 & 0.113 & 0.114 & 0.498 & 0.240 & 0.176 & 0.442 \\
-0.146 & -0.110 & -0.103 & -0.012 & -0.113 & -0.066 & -0.030 & -0.025 \\
1.208 & 0.294 & 0.264 & 0.177 & 0.665 & 0.364 & 0.267 & 0.533 \\
3.973 & 1.719 & 2.297 & 2.301 & 2.440 & 2.310 & 2.419 & 2.297 \\
2.053 & 2.051 & 2.068 & 2.043 & 1.924 & 1.961 & 1.973 & 2.031 \\
0.255 & 0.079 & 0.049 & 0.050 & 0.204 & 0.104 & 0.073 & 0.193 \\
$58.33 \%$ & $61.02 \%$ & $55.17 \%$ & $56.36 \%$ & $59.62 \%$ & $57.14 \%$ & $56.52 \%$ & $58.14 \%$ \\
$2.215^{*}$ & 0.939 & 0.671 & 0.448 & 1.520 & 0.814 & 0.571 & 1.099 \\
$2.115^{*}$ & 1.015 & 1.138 & 0.720 & $1.749 *$ & 1.197 & 0.882 & 1.481 \\
\hline
\end{tabular}

U.K. $\quad$ Mean Ret.(\%) - Active Strategy

Mean Ret.(\%) - Passive Equal Weighted Portfolio

Excess Return (\%)

Std. Dev.(\%) - Active Strategy

Std. Dev.(\%) - Passive Equal Weighted Portfolio

Infor. Ratio

Prob $>$ Passive Equal Weighted Portfolio (\%)

: paired t-test

: Wilcoxon test

\begin{tabular}{cccccccc}
1.323 & 0.472 & 0.285 & 0.007 & 0.209 & 0.190 & -0.004 & 0.180 \\
-0.188 & -0.211 & -0.194 & -0.221 & -0.218 & -0.256 & -0.264 & -0.351 \\
1.511 & 0.683 & 0.479 & 0.228 & 0.427 & 0.446 & 0.259 & 0.531 \\
3.893 & 1.985 & 2.289 & 2.437 & 2.051 & 2.064 & 2.095 & 2.356 \\
1.860 & 1.868 & 1.879 & 1.912 & 1.930 & 1.961 & 1.987 & 1.958 \\
0.340 & 0.238 & 0.124 & 0.003 & 0.102 & 0.092 & -0.002 & 0.076 \\
$70.00 \%$ & $64.41 \%$ & $55.17 \%$ & $52.73 \%$ & $61.54 \%$ & $53.06 \%$ & $52.17 \%$ & $60.47 \%$ \\
$2.701^{* *}$ & 1.711 & 1.283 & 0.442 & 1.043 & 0.998 & 0.483 & 1.078 \\
$2.556^{* *}$ & $2.064^{*}$ & 1.287 & 0.756 & 1.131 & 0.849 & 1.070 & 1.170 \\
\hline
\end{tabular}

U.S.A. Mean Ret.(\%) - Active Strategy

\begin{tabular}{cccccccc}
0.618 & -0.169 & -0.105 & -0.089 & 0.119 & -0.110 & -0.147 & -0.061 \\
0.208 & 0.244 & 0.255 & 0.291 & 0.208 & 0.231 & 0.285 & 0.363 \\
0.414 & -0.410 & -0.357 & -0.376 & -0.086 & -0.337 & -0.429 & -0.420 \\
4.092 & 1.616 & 2.062 & 2.330 & 2.336 & 2.387 & 2.297 & 2.474 \\
1.815 & 1.808 & 1.822 & 1.860 & 1.873 & 1.916 & 1.915 & 1.882 \\
0.151 & -0.105 & -0.051 & -0.038 & 0.051 & -0.046 & -0.064 & -0.025 \\
$51.67 \%$ & $40.68 \%$ & $48.28 \%$ & $43.64 \%$ & $48.08 \%$ & $44.90 \%$ & $43.48 \%$ & $46.51 \%$ \\
0.821 & -1.400 & -0.947 & -0.925 & -0.203 & -0.761 & -0.982 & -0.942 \\
0.436 & -1.181 & -0.801 & -0.930 & -0.208 & -0.821 & -1.046 & -0.903 \\
\hline
\end{tabular}

Mean Ret.(\%) - Passive Equal Weighted Portfolio

Excess Return (\%)

Std. Dev.(\%) - Active Strategy

Std. Dev.(\%) - Passive Equal Weighted Portfolio

Infor. Ratio

Prob > Passive Equal Weighted Portfolio (\%)

: paired t-test

: Wilcoxon test

\begin{tabular}{lllllllll}
0.436 & -1.181 & -0.801 & -0.930 & -0.208 & -0.821 & -1.046 & -0.903 \\
\hline
\end{tabular} 


\begin{tabular}{|c|c|c|c|c|c|c|c|c|c|}
\hline \multirow[t]{9}{*}{ JAPAN } & Mean Ret.(\%) - Active Strategy & 0.996 & 0.153 & -0.299 & 0.145 & 0.393 & 0.238 & 0.226 & 0.420 \\
\hline & Mean Ret.(\%) - Passive Equal Weighted Portfolio & 0.335 & 0.318 & 0.328 & 0.305 & 0.513 & 0.631 & 0.665 & 0.649 \\
\hline & Excess Return (\%) & 0.637 & -0.189 & -0.651 & -0.186 & -0.147 & -0.422 & -0.469 & -0.262 \\
\hline & Std. Dev.(\%) - Active Strategy & 3.747 & 1.852 & 2.859 & 2.042 & 1.961 & 2.037 & 2.131 & 2.295 \\
\hline & Std. Dev.(\%) - Passive Equal Weighted Portfolio & 2.736 & 2.756 & 2.779 & 2.848 & 2.769 & 2.769 & 2.592 & 2.644 \\
\hline & Infor. Ratio & 0.266 & 0.083 & -0.104 & 0.071 & 0.200 & 0.117 & 0.106 & 0.183 \\
\hline & Prob > Passive Equal Weighted Portfolio (\%) & $56.67 \%$ & $54.24 \%$ & $50.00 \%$ & $52.73 \%$ & $53.85 \%$ & $40.82 \%$ & $41.30 \%$ & $51.16 \%$ \\
\hline & : paired t-test & 1.111 & -0.412 & -1.214 & -0.350 & -0.286 & -0.789 & -0.886 & -0.470 \\
\hline & : Wilcoxon test & 1.102 & -0.180 & -0.889 & 0.027 & 0.150 & -0.565 & -0.515 & 0.004 \\
\hline \multirow[t]{9}{*}{ EURO } & Mean Ret.(\%) - Active Strategy & 0.310 & -0.197 & 0.083 & 0.049 & 0.066 & 0.199 & -0.027 & -0.043 \\
\hline & Mean Ret.(\%) - Passive Equal Weighted Portfolio & 0.002 & -0.031 & -0.076 & -0.152 & -0.191 & -0.349 & -0.481 & -0.470 \\
\hline & Excess Return (\%) & 0.329 & -0.145 & 0.180 & 0.223 & 0.281 & 0.572 & 0.481 & 0.455 \\
\hline & Std. Dev.(\%) - Active Strategy & 3.526 & 1.894 & 2.545 & 2.221 & 1.997 & 2.058 & 2.015 & 2.072 \\
\hline & Std. Dev.(\%) - Passive Equal Weighted Portfolio & 2.399 & 2.406 & 2.402 & 2.434 & 2.461 & 2.422 & 2.403 & 2.402 \\
\hline & Infor. Ratio & 0.088 & -0.104 & 0.033 & 0.022 & 0.033 & 0.097 & -0.013 & -0.021 \\
\hline & Prob > Passive Equal Weighted Portfolio (\%) & $58.33 \%$ & $47.46 \%$ & $50.00 \%$ & $52.73 \%$ & $53.85 \%$ & $53.06 \%$ & $54.35 \%$ & $55.81 \%$ \\
\hline & :paired t-test & 0.533 & -0.348 & 0.371 & 0.480 & 0.642 & 1.228 & 1.060 & 0.990 \\
\hline & :Wilcoxon test & 0.772 & -0.390 & 0.133 & 0.547 & 0.644 & 1.126 & 1.062 & 1.015 \\
\hline
\end{tabular}

Table 8 presents the results of Strategy Two back tested over various formation look back periods (from 1 to 18 months) utlising raw currency returns as the source of momentum. Excess return refers to profits generated by the strategy which are greater than the passive buy-and-hold equal weighted portfolio of the respective six foreign currencies. The parametric paired t-test and the non-parametric Wilcoxon test is shown to test the statistical significance of the excess returns relative to the passive equal weighted long only portfolio, ** and * indicate statistical significance at the $1 \%$ and $5 \%$ levels, respectively. 
TABLE 8

Strategy Two Results - 1999 to 2004

Panel B: Source of Momentum - Interest Adjusted Returns

Momentum Formation Period (in months):

12

CANADA Mean Ret.(\%) - Active Strategy

Mean Ret.(\%) - Passive Equal Weighted Portfolio

Excess Return (\%)

Std. Dev.(\%) - Active Strategy

Std. Dev.(\%) - Passive Equal Weighted Portfolio Infor. Ratio

Prob > Passive Equal Weighted Portfolio (\%)

: paired t-test

: Wilcoxon test

\begin{tabular}{cccccccc}
0.968 & 0.380 & 0.224 & 0.370 & 1.057 & 1.387 & 0.765 & 1.433 \\
-0.146 & -0.110 & -0.103 & -0.012 & -0.113 & -0.066 & -0.030 & -0.025 \\
1.114 & 0.491 & 0.326 & 0.381 & 1.170 & 1.453 & 0.795 & 1.458 \\
3.881 & 3.584 & 3.601 & 4.208 & 4.400 & 4.137 & 5.103 & 5.023 \\
2.053 & 2.051 & 2.068 & 2.043 & 1.924 & 1.961 & 1.973 & 2.031 \\
0.249 & 0.106 & 0.062 & 0.088 & 0.240 & 0.335 & 0.150 & 0.285 \\
$63.33 \%$ & $57.63 \%$ & $62.07 \%$ & $56.36 \%$ & $61.54 \%$ & $65.31 \%$ & $56.52 \%$ & $60.47 \%$ \\
1.836 & 0.826 & 0.550 & 0.551 & 1.968 & 2.208 & 0.993 & 1.728 \\
$1.974 *$ & 0.692 & 0.784 & 0.266 & $2.301 *$ & $2.228^{*}$ & 1.242 & $1.922^{*}$ \\
\hline
\end{tabular}

U.K. $\quad$ Mean Ret.(\%) - Active Strategy

Mean Ret.(\%) - Passive Equal Weighted Portfolio

Excess Return (\%)

Std. Dev.(\%) - Active Strategy

Std. Dev.(\%) - Passive Equal Weighted Portfolio

Infor. Ratio

Prob > Passive Equal Weighted Portfolio (\%)

: paired t-test

: Wilcoxon test

\begin{tabular}{cccccccc}
1.385 & 1.121 & 0.453 & 0.170 & 0.655 & 0.323 & 0.957 & 0.742 \\
-0.188 & -0.211 & -0.194 & -0.221 & -0.218 & -0.256 & -0.264 & -0.351 \\
1.572 & 1.333 & 0.647 & 0.391 & 0.873 & 0.579 & 1.220 & 1.093 \\
4.016 & 4.018 & 4.496 & 4.353 & 4.156 & 3.830 & 4.161 & 4.691 \\
1.860 & 1.868 & 1.879 & 1.912 & 1.930 & 1.961 & 1.987 & 1.958 \\
0.880 & 0.842 & 0.700 & 0.434 & 0.750 & 0.558 & 0.784 & 0.679 \\
$63.33 \%$ & $59.32 \%$ & $53.45 \%$ & $52.73 \%$ & $61.54 \%$ & $51.02 \%$ & $60.87 \%$ & $55.81 \%$ \\
$2.706^{* *}$ & $2.278^{*}$ & 0.967 & 0.579 & 1.403 & 0.862 & 1.679 & 1.354 \\
$3.023^{* *}$ & $2.554^{* *}$ & 1.071 & 0.601 & 1.495 & 0.828 & $1.804 *$ & $1.732^{*}$ \\
\hline & & & & & & & \\
0.775 & 0.107 & 0.760 & -0.075 & 0.687 & 0.338 & -0.010 & 0.350 \\
0.208 & 0.244 & 0.255 & 0.291 & 0.208 & 0.231 & 0.285 & 0.363 \\
0.568 & -0.137 & 0.505 & -0.365 & 0.479 & 0.106 & -0.296 & -0.013 \\
3.958 & 3.570 & 4.339 & 4.496 & 4.415 & 4.270 & 4.944 & 4.963 \\
1.815 & 1.808 & 1.822 & 1.860 & 1.873 & 1.916 & 1.915 & 1.882 \\
0.196 & 0.030 & 0.175 & -0.017 & 0.156 & 0.079 & -0.002 & 0.071 \\
$55.00 \%$ & $52.54 \%$ & $55.17 \%$ & $47.27 \%$ & $57.69 \%$ & $51.02 \%$ & $47.83 \%$ & $51.16 \%$ \\
1.085 & -0.276 & 0.813 & -0.555 & 0.768 & 0.164 & -0.363 & -0.016 \\
0.971 & 0.030 & 0.674 & -0.995 & 0.702 & -0.203 & -0.531 & -0.186 \\
\hline
\end{tabular}

U.S.A. Mean Ret.(\%) - Active Strategy

Mean Ret.(\%) - Passive Equal Weighted Portfolio

Excess Return (\%)

Std. Dev.(\%) - Active Strategy

Std. Dev.(\%) - Passive Equal Weighted Portfolio

Infor. Ratio

Prob > Passive Equal Weighted Portfolio (\%)

: paired t-test

: Wilcoxon test

0.971 


\begin{tabular}{|c|c|c|c|c|c|c|c|c|c|}
\hline \multirow[t]{9}{*}{ JAPAN } & Mean Ret.(\%) - Active Strategy & 0.927 & 0.357 & 0.063 & 0.488 & 1.173 & 0.618 & 0.212 & 0.969 \\
\hline & Mean Ret.(\%) - Passive Equal Weighted Portfolio & 0.335 & 0.318 & 0.328 & 0.305 & 0.513 & 0.631 & 0.665 & 0.649 \\
\hline & Excess Return (\%) & 0.592 & 0.038 & -0.265 & 0.183 & 0.660 & -0.013 & -0.453 & 0.320 \\
\hline & Std. Dev.(\%) - Active Strategy & 3.709 & 3.682 & 4.037 & 4.199 & 4.293 & 4.087 & 4.517 & 4.398 \\
\hline & Std. Dev.(\%) - Passive Equal Weighted Portfolio & 2.736 & 2.756 & 2.779 & 2.848 & 2.769 & 2.769 & 2.592 & 2.644 \\
\hline & Infor. Ratio & 0.250 & 0.097 & 0.016 & 0.116 & 0.273 & 0.151 & 0.047 & 0.220 \\
\hline & Prob > Passive Equal Weighted Portfolio (\%) & $51.67 \%$ & $47.46 \%$ & $48.28 \%$ & $52.73 \%$ & $63.46 \%$ & $53.06 \%$ & $45.65 \%$ & $48.84 \%$ \\
\hline & : paired t-test & 1.035 & 0.065 & -0.433 & 0.269 & 0.971 & -0.018 & -0.581 & 0.397 \\
\hline & : Wilcoxon test & 1.029 & 0.250 & -0.409 & 0.332 & 0.943 & 0.266 & -0.328 & 0.730 \\
\hline \multirow[t]{9}{*}{ EURO } & Mean Ret.(\%) - Active Strategy & 0.371 & -0.078 & 0.168 & 0.393 & -0.188 & 0.562 & 0.964 & 0.551 \\
\hline & Mean Ret.(\%) - Passive Equal Weighted Portfolio & 0.002 & -0.031 & -0.076 & -0.152 & -0.191 & -0.349 & -0.481 & -0.470 \\
\hline & Excess Return (\%) & 0.369 & -0.047 & 0.243 & 0.545 & 0.003 & 0.910 & 1.445 & 1.021 \\
\hline & Std. Dev.(\%) - Active Strategy & 3.777 & 3.560 & 3.719 & 4.062 & 4.265 & 3.888 & 3.858 & 4.305 \\
\hline & Std. Dev.(\%) - Passive Equal Weighted Portfolio & 2.399 & 2.406 & 2.402 & 2.434 & 2.461 & 2.422 & 2.403 & 2.402 \\
\hline & Infor. Ratio & 0.098 & -0.022 & 0.045 & 0.097 & -0.044 & 0.144 & 0.250 & 0.128 \\
\hline & Prob > Passive Equal Weighted Portfolio (\%) & $56.67 \%$ & $45.76 \%$ & $46.55 \%$ & $52.73 \%$ & $46.15 \%$ & $48.98 \%$ & $56.52 \%$ & $53.49 \%$ \\
\hline & :paired t-test & 0.671 & -0.085 & 0.423 & 0.785 & 0.006 & 1.326 & $2.021^{*}$ & 1.432 \\
\hline & :Wilcoxon test & 1.139 & 0.035 & 0.718 & 0.714 & 0.039 & 1.247 & $2.015^{*}$ & 1.533 \\
\hline
\end{tabular}

Table 8 presents the results of Strategy Two back tested over various formation look back periods (from 1 to 18 months) utlising raw currency returns as the source of momentum. Excess return refers to profits generated by the strategy which are greater than the passive buy-and-hold equal weighted portfolio of the respective six foreign currencies. The parametric paired t-test and the non-parametric Wilcoxon test is shown to test the statistical significance of the excess returns relative to the passive equal weighted long only portfolio, ** and $*$ indicate statistical significance at the $1 \%$ and $5 \%$ levels, respectively. 
TABLE 9

Best Two Momentum Strategies For Each Currency

And The Impact of Transaction Costs

1980 to 1998

\begin{tabular}{ccc}
\hline COUNTRY & $\begin{array}{c}\text { STRATEGY } \\
\text { DETAILS }\end{array}$ & STATISTICAL \\
DIAGNOSTICS
\end{tabular}

ITALY STRATEGY 2 Excess Return (\%) after T.Costs

L2/S2 Prob > Passive Equal Weighted Portfolio (\%)

12 Months :paired t-test

:Wilcoxon test

U.K. STRATEGY 1 Excess Return (\%) after T.Costs

L1/S1 Prob > Passive Equal Weighted Portfolio (\%)

12 Months :paired t-test

:Wilcoxon test

U.K. STRATEGY 2 Excess Return (\%) after T.Costs

L2/S2 Prob $>$ Passive Equal Weighted Portfolio (\%)

12 Months :paired t-test

:Wilcoxon test

U.S.A. STRATEGY 1 Excess Return (\%) after T.Costs

L1/S1 Prob > Passive Equal Weighted Portfolio (\%)

6 Months

:paired t-test

:Wilcoxon test

U.S.A. STRATEGY 1 Excess Return (\%) after T.Costs

L1/S1 Prob > Passive Equal Weighted Portfolio (\%)

3 Months

:paired t-test

:Wilcoxon test

CANADA STRATEGY 1 Excess Return (\%) after T.Costs

L1/S1 Prob > Passive Equal Weighted Portfolio (\%)

1 Month

:paired t-test

:Wilcoxon test

CANADA STRATEGY 1 Excess Return (\%) after T.Costs

L1/S1 Prob > Passive Equal Weighted Portfolio (\%)

3 Months

:paired t-test

:Wilcoxon test

\begin{tabular}{|c|c|c|c|c|c|}
\hline \multicolumn{6}{|c|}{ No. of Ticks From Mid Point multiplied by four } \\
\hline 0 & 5 & 10 & 15 & 20 & 25 \\
\hline 0.907 & 0.769 & 0.632 & 0.494 & 0.356 & 0.219 \\
\hline $61.61 \%$ & $61.14 \%$ & $60.19 \%$ & $60.19 \%$ & $59.72 \%$ & $55.45 \%$ \\
\hline $3.337 * *$ & $2.830 * *$ & $2.322^{*}$ & 1.816 & 1.309 & 0.803 \\
\hline $4.620 * *$ & $4.118^{* *}$ & $3.562 * *$ & $3.025 * *$ & $2.476^{* *}$ & $1.904 *$ \\
\hline 0.584 & 0.447 & 0.310 & 0.174 & 0.037 & -0.100 \\
\hline $67.80 \%$ & $66.34 \%$ & $62.44 \%$ & $61.46 \%$ & $59.02 \%$ & $57.07 \%$ \\
\hline $2.330 *$ & 1.783 & 1.238 & 0.692 & 0.148 & -0.397 \\
\hline $4.792^{* *}$ & $4.254^{* *}$ & $3.673^{* *}$ & $3.063^{* *}$ & $2.429 * *$ & 1.813* \\
\hline 0.570 & 0.249 & -0.072 & -0.393 & -0.714 & -1.035 \\
\hline $65.85 \%$ & $56.59 \%$ & $54.63 \%$ & $51.22 \%$ & $48.29 \%$ & $45.37 \%$ \\
\hline $2.272^{*}$ & 0.752 & -0.216 & -1.182 & -2.145 & -3.106 \\
\hline $4.388 * *$ & $2.523 * *$ & 1.398 & 0.245 & -0.872 & -1.987 \\
\hline 0.451 & 0.130 & -0.191 & -0.512 & -0.833 & -1.154 \\
\hline $59.02 \%$ & $56.59 \%$ & $53.66 \%$ & $49.27 \%$ & $43.90 \%$ & $39.02 \%$ \\
\hline $2.330 *$ & 0.467 & -0.684 & -1.834 & $-2.981 * *$ & $-4.126^{* *}$ \\
\hline $4.792 * *$ & $1.758 *$ & 0.400 & -0.963 & $-2.404^{* *}$ & $-3.869 * *$ \\
\hline 0.429 & 0.274 & 0.118 & -0.038 & -0.193 & -0.349 \\
\hline $53.08 \%$ & $52.13 \%$ & $51.66 \%$ & $51.18 \%$ & $48.34 \%$ & $46.45 \%$ \\
\hline 1.484 & 0.946 & 0.408 & -0.130 & -0.668 & -1.206 \\
\hline $2.228^{*}$ & $1.672 *$ & 1.128 & 0.278 & 0.007 & -0.557 \\
\hline 0.491 & 0.335 & 0.179 & 0.023 & -0.132 & -0.288 \\
\hline $55.14 \%$ & $52.80 \%$ & $50.93 \%$ & $48.13 \%$ & $46.26 \%$ & $44.86 \%$ \\
\hline 1.600 & 1.093 & 0.584 & 0.076 & -0.432 & -0.941 \\
\hline $2.021^{*}$ & 1.490 & 0.982 & 0.449 & -0.112 & -0.678 \\
\hline 0.673 & 0.466 & 0.310 & 0.154 & -0.002 & -0.157 \\
\hline $58.33 \%$ & $57.94 \%$ & $55.14 \%$ & $54.67 \%$ & $53.27 \%$ & $50.93 \%$ \\
\hline $2.290 *$ & 1.588 & 1.057 & 0.526 & -0.006 & -0.538 \\
\hline $2.854 * *$ & $2.315^{*}$ & $1.783^{*}$ & 1.195 & 0.635 & 0.067 \\
\hline 0.561 & 0.480 & 0.399 & 0.318 & 0.237 & 0.156 \\
\hline $58.41 \%$ & $58.41 \%$ & $57.01 \%$ & $55.14 \%$ & $54.67 \%$ & $54.21 \%$ \\
\hline 1.896998 & 1.623 & 1.349 & 1.075 & 0.801 & 0.527 \\
\hline $2.678 * *$ & $2.398 * *$ & $2.113^{*}$ & $1.834 *$ & 1.558 & 1.262 \\
\hline
\end{tabular}


JAPAN STRATEGY 1 Excess Return (\%) after T.Costs

L1/S1

6 Months

$$
\text { :paired t-test }
$$

Prob > Passive Equal Weighted Portfolio (\%)

:Wilcoxon test

JAPAN STRATEGY 1 Excess Return (\%) after T.Costs

L1/S1

3 Months

Prob > Passive Equal Weighted Portfolio (\%)

$$
\text { :paired t-test }
$$

:Wilcoxon test

GERMANY STRATEGY 1 Excess Return (\%) after T.Costs

L1/S1

Prob > Passive Equal Weighted Portfolio (\%)

6 Months

:paired t-test

:Wilcoxon test

GERMANY STRATEGY 1 Excess Return (\%) after T.Costs

L1/S1 Prob $>$ Passive Equal Weighted Portfolio (\%)

12 Months
:Wilcoxon test

FRANCE STRATEGY 1 Excess Return (\%) after T.Costs

L1/S1 Prob > Passive Equal Weighted Portfolio (\%)

6 Months

:paired t-test

:Wilcoxon test

FRANCE STRATEGY 1 Excess Return (\%) after T.Costs

L1/S1 Prob > Passive Equal Weighted Portfolio (\%)

3 Months :paired t-test

\begin{tabular}{|c|c|c|c|c|c|}
\hline 0.348 & 0.207 & 0.065 & -0.076 & -0.218 & -0.359 \\
\hline $51.18 \%$ & $48.82 \%$ & $47.87 \%$ & $47.39 \%$ & $46.92 \%$ & $44.08 \%$ \\
\hline 1.095 & 0.650 & 0.205 & -0.239 & -0.683 & -1.127 \\
\hline 1.505 & 1.006 & 0.320 & -0.061 & -0.545 & -1.023 \\
\hline 0.454 & 0.313 & 0.173 & 0.032 & -0.108 & -0.248 \\
\hline $50.47 \%$ & $49.07 \%$ & $47.66 \%$ & $47.20 \%$ & $46.26 \%$ & $44.39 \%$ \\
\hline 1.444 & 0.996 & 0.549 & 0.103 & -0.342 & -0.785 \\
\hline 1.496 & 0.999 & 0.477 & -0.034 & -0.542 & -1.023 \\
\hline 0.665 & 0.557 & 0.449 & 0.341 & 0.233 & 0.126 \\
\hline $57.35 \%$ & $56.87 \%$ & $54.98 \%$ & $54.98 \%$ & $53.55 \%$ & $52.61 \%$ \\
\hline 2.398* & $2.008 *$ & 1.618 & 1.229 & 0.841 & 0.452 \\
\hline $3.444 * *$ & $3.057 * *$ & $2.627 * *$ & $2.222 *$ & 1.793* & 1.415 \\
\hline 0.323 & 0.214 & 0.106 & -0.003 & -0.111 & -0.220 \\
\hline $59.02 \%$ & $57.07 \%$ & $54.63 \%$ & $54.15 \%$ & $53.17 \%$ & $52.68 \%$ \\
\hline 1.059 & 0.703 & 0.347 & -0.009 & -0.365 & -0.721 \\
\hline $3.007 * *$ & $2.630 * *$ & $2.217 *$ & $1.808 *$ & 1.427 & 1.008 \\
\hline 0.725 & 0.692 & 0.659 & 0.626 & 0.593 & 0.560 \\
\hline $63.51 \%$ & $62.56 \%$ & $62.09 \%$ & $62.09 \%$ & $60.66 \%$ & $60.19 \%$ \\
\hline $2.613^{* *}$ & $2.494 * *$ & $2.375 * *$ & $2.256^{*}$ & 2.137* & $2.018 *$ \\
\hline $4.182 * *$ & $4.056 * *$ & $3.933 * *$ & $3.817 * *$ & $3.698 * *$ & $3.586 * *$ \\
\hline 0.744 & 0.711 & 0.678 & 0.645 & 0.612 & 0.579 \\
\hline $61.68 \%$ & $60.75 \%$ & $60.28 \%$ & $59.81 \%$ & $58.88 \%$ & $58.41 \%$ \\
\hline $2.784 * *$ & $2.660 * *$ & 2.536* & $2.412 *$ & 2.288* & $2.165 *$ \\
\hline $3.631 * *$ & $3.505^{* *}$ & $3.386 * *$ & $3.254 * *$ & $3.116^{* *}$ & $3.000 * *$ \\
\hline
\end{tabular}

:Wilcoxon test
Table 9 estimates the impact of transaction costs on the best two momentum strategies of each currency in the 1980 to 1998 period. These estimates assume four transactions occur at the end of each month, that is, the two currencies open are closed and another two currencies are entered into in order to construct the following month's currency long/short exposure. 
TABLE 10

Performance of Strategy One with Consolidated Look back Rankings Source of Momentum: Interest Adjusted Returns

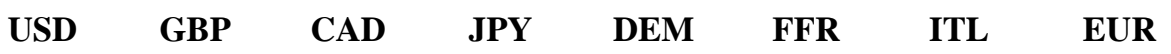

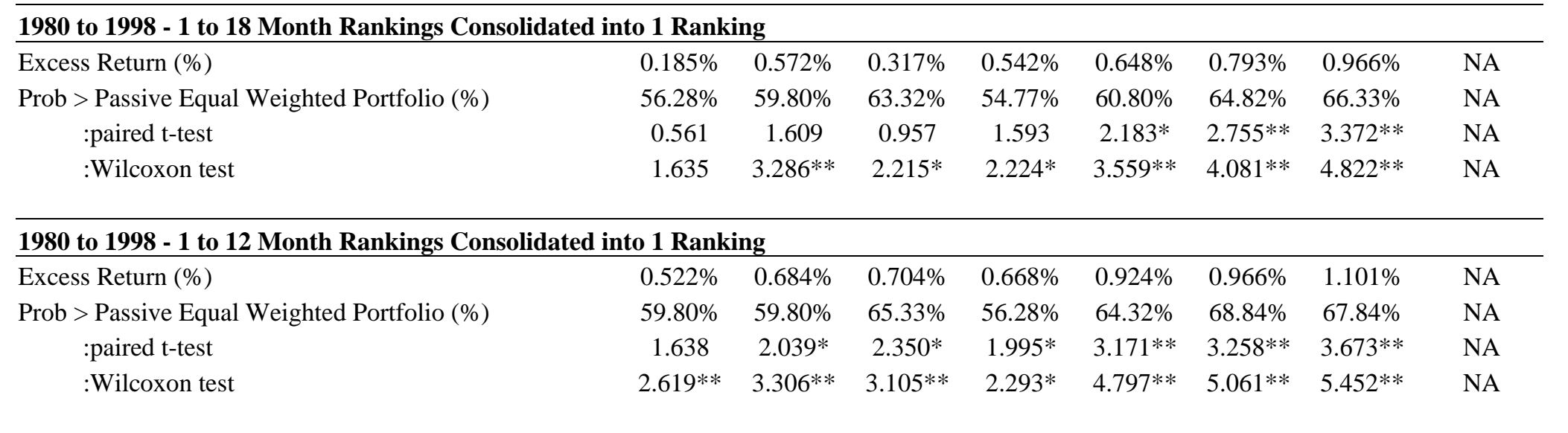

\section{9 to 2004 - 1 to 18 Month Rankings Consolidated into 1 Ranking}

\begin{tabular}{ccccccccc}
\hline Excess Return (\%) & $1.900 \%$ & $2.833 \%$ & $2.360 \%$ & $1.259 \%$ & NA & NA & NA & $2.779 \%$ \\
Prob > Passive Equal Weighted Portfolio (\%) & $72.73 \%$ & $79.07 \%$ & $70.45 \%$ & $72.09 \%$ & NA & NA & NA & $79.070 \%$ \\
$\quad$ :paired t-test & $3.558^{* *}$ & $5.092^{* *}$ & $4.304^{* *}$ & 1.334 & NA & NA & NA & $4.470^{* *}$ \\
$\quad$ :Wilcoxon test & $3.278^{* *}$ & $5.057^{* *}$ & $3.922^{* *}$ & $3.174^{* *}$ & NA & NA & NA & $4.444^{* *}$
\end{tabular}

\section{9 to 2004 - 1 to 12 Month Rankings Consolidated into 1 Ranking}

Excess Return (\%)

Prob > Passive Equal Weighted Portfolio (\%)

:paired t-test

:Wilcoxon test

$\begin{array}{llllllll}2.422 \% & 3.008 \% & 2.326 \% & 1.810 \% & \text { NA } & \text { NA } & \text { NA } & 2.860 \% \\ 79.59 \% & 77.55 \% & 70.45 \% & 63.27 \% & \text { NA } & \text { NA } & \text { NA } & 85.714 \% \\ 5.909 * * & 5.336 * * & 4.272 * * & 3.833 * * & \text { NA } & \text { NA } & \text { NA } & 5.198 * * \\ 4.707 * * & 5.446 * * & 5.191 * * & 3.663 * * & \text { NA } & \text { NA } & \text { NA } & 4.999 * *\end{array}$

Table 10 presents the results of Strategy One with the rankings of all the various look back periods consolidated into one ranking set. The active strategy's excess return were measured against a passive long only equal weighted portfolio of the respective six foreign currencies. The statistical significance of excess returns was evaluated using the paired t-test and the non-parametric Wilcoxon test. The results in Table 10 are free of transaction costs, ** and * indicate statistical significance at the 1\% and 5\% levels, respectively. 


\section{TABLE 11 \\ Bootstrap Simulation Results \\ Strategy One \\ Source of Momentum: Interest Adjusted Returns}

\begin{tabular}{|c|c|c|c|c|c|c|c|c|}
\hline & USD & GBP & CAD & JPY & DEM & FFR & ITL & EUR \\
\hline \multicolumn{9}{|c|}{1980 to 1998 - 1 to 18 Month Rankings Consolidated into 1 Ranking } \\
\hline Excess Return (\%) Average & $-0.090 \%$ & $0.013 \%$ & $0.010 \%$ & $-0.045 \%$ & $-0.112 \%$ & $0.019 \%$ & $0.150 \%$ & NA \\
\hline Information Ratio & -0.023 & 0.004 & 0.002 & -0.012 & -0.029 & 0.005 & 0.039 & NA \\
\hline Prob Active Strategy > Passive Portfolio & $49.70 \%$ & $51.08 \%$ & $51.01 \%$ & $48.06 \%$ & $49.32 \%$ & $51.32 \%$ & $53.07 \%$ & NA \\
\hline \multicolumn{9}{|c|}{1980 to 1998 - 1 to 12 Month Rankings Consolidated into 1 Ranking } \\
\hline Excess Return (\%) Average & $-0.089 \%$ & $0.013 \%$ & $0.008 \%$ & $-0.046 \%$ & $-0.115 \%$ & $0.017 \%$ & $0.143 \%$ & NA \\
\hline Information Ratio & -0.022 & 0.004 & 0.002 & -0.012 & -0.030 & 0.005 & 0.038 & NA \\
\hline Prob Active Strategy > Passive Portfolio & $49.75 \%$ & $51.05 \%$ & $50.99 \%$ & $48.03 \%$ & $49.31 \%$ & $51.31 \%$ & $53.00 \%$ & NA \\
\hline \multicolumn{9}{|c|}{1999 to 2004 - 1 to 18 Month Rankings Consolidated into 1 Ranking } \\
\hline Excess Return (\%) Average & $-0.004 \%$ & $0.325 \%$ & $0.350 \%$ & $-0.311 \%$ & NA & NA & NA & $0.169 \%$ \\
\hline Information Ratio & -0.001 & 0.096 & 0.102 & -0.084 & NA & NA & NA & 0.046 \\
\hline Prob Active Strategy > Passive Portfolio & $50.86 \%$ & $54.28 \%$ & $55.69 \%$ & $47.02 \%$ & NA & NA & NA & $51.92 \%$ \\
\hline \multicolumn{9}{|c|}{1999 to 2004 - 1 to 12 Month Rankings Consolidated into 1 Ranking } \\
\hline Excess Return (\%) Average & $-0.017 \%$ & $0.305 \%$ & $0.337 \%$ & $-0.317 \%$ & NA & NA & NA & $0.159 \%$ \\
\hline Information Ratio & -0.004 & 0.090 & 0.098 & -0.085 & NA & NA & NA & 0.044 \\
\hline Prob Active Strategy > Passive Portfolio & $50.66 \%$ & $53.82 \%$ & $55.52 \%$ & $46.79 \%$ & NA & NA & NA & $51.95 \%$ \\
\hline
\end{tabular}

Table 11 illustrates the summary results based on 1,000 bootstrap simulations that replicate the 1980 to 1998 period and the 1999 to 2004 period. The bootstrap replications were sourced from the original dataset, however, the assumption of i.i.d. returns is imposed whereby any autocorrelation structure in the time series is ignored. The simulations are based on zero transaction costs. 


\section{LISTING OF DISCUSSION PAPERS - 2003}

Valadkhani A, History of Macroeconomic Modelling: Lessons from Past Experience, No 131, January 2003

Valadkhani A, Long and Short-Run Determinants of Money Demand in New Zealand: Evidence from Co Integration Analysis, No 132

January 2003

Anderson J, Optimal F and Portfolio Return Optimisation in US Futures Markets, No 133, January 2003

Anderson J, A Test of Weak-Form Market Efficiency in Australia Bank Bil Futures Calendar Spreads, No 134, January 2003

Aruman S, The Effectiveness of Foreign Exchange Intervention in Australia: A Factor Model Approach with GARCH Specifications, No 135 January 2003

Lahiri R, A Further Exploration of Some Computational Issues in Equilibrium Business Cycle Theory, No 136, February 2003

Valadkhani A, How Many Jobs Were Lost With the Collapse of Ansett? No 137, February 2003

Drew M E, Naughton T, \& Veerarghavan M, Is Idiosyncratic Volatility Priced? Evidence from the Shanghai Stock Exchange, No 138, February 2003

Valadkhani, A, Does the Term Structure Predict Australia's Future Output Growth? No 139, February 2003

Worthington $\mathrm{A}$, \& Higgs $\mathrm{H}$, A Multivariate GARCH Analysis of the Domestic Transmission of Energy Commodity Prices and Volatility: A Comparison of the Peak and Off-peak Periods in the Australian Electricity Spot Market, No 140, February 2003

Li S, The Estimation of Implied Volatility from the Black-Scholes Model: Some New Formulas and Their Applications, No 141, February 2003
Drew M E, \& Stanford, J D, Principal and Agent Problems in Superannuation Funds, No 142, March 2003

Li S, A Single-Period Model and Some Empirical Evidences for Optimal Asset Allocation with Value-at-Risk Constraints, No 143, March 2003

Valadkhani A, An Empirical Analysis of the Black Market Exchange Rate in Iran, No 144, April 2003

Worthington A, Business Expectations and Preferences regarding the Introduction of Daylight Saving in Queensland, No 145, May 2003

Worthington A, Losing Sleep at the Market: An Empirical Note on the Daylight Saving Anomaly in Australia, No 146, May 2003

Robinson M, Tightening the Results/Funding Link in Performance Budgeting Systems, No 147, May 2003

Worthington A, \& Higgs $\mathrm{H}$, Risk, Return and Portfolio Diversification in Major Painting Marketing: The Application of Conventional Financial Analysis to Unconventional Investments, No 148, June 2003

Valadkhani A, Demand for M2 in Developing Countries: An Empirical Panel Investigation, No 149, July 2003

Worthington A, \& Higgs $\mathrm{H}$, Modelling the Intraday Return Volatility Process in the Australia Equity Market: An Examination of the Role of Information Arrival in S \& PASX Stocks, No 150, July 2003

Lahiri R, Tax Distortions in a Neoclassical Monetary Economy in the Presence of Administration Costs, No 151 September 2003

Layton A, \& Smith D, Duration Dependence in the US Business Cycle, No 152, August 2003

Valadkhani A, \& Layton A, Quantifying the Effect of GST on Inflation in Australia's Capital Cities: An Intervention Analysis, No 153, September 2003 
Worthington A, \& Valadkhani A, Measuring the Impact of Natural Disasters on Capital Markets: An Empirical Application Using Intervention Analysis, No 154, September 2003

Robinson M, The Output Concept and Public Sector Services, No 155, September 2003

Worthington A, Brown K, Crawford M, \& Pickernell D, Socio-Economic and Demographic Determinants of Household Gambling in Australia, No 156, September 2003

Worthington A, \& Higgs $\mathrm{H}$, Tests of Random Walks and Market Efficiency in Latin American Stock Markets: An Empirical Note, No 157, September 2003

(Replacing Previous No 158) Worthington A, \& Higgs H, Systematic Features of High-Frequency Volatility in Australian Electricity Markets: Intraday Patterns, Information Arrival and Calendar Effects, No 158, November 2003

Worthington A, \& Higgs $\mathrm{H}$, Weak-form Market Efficiency in European Emerging and Developed Stock Markets, No 159, September 2003

West T, \& Worthington A, Macroeconomic Risk Factors in Australian Commercial Real Estate, Listed Property Trust and Property Sector Stock Returns: A Comparative Analysis using GARCH-M, No 160, October 2003

Lee B, Interstate Comparison of Output and Productivity in the Australian Agricultural Sector - 1991 - 1999, No 161, October 2003

McCarthy S, Hedging Versus not Hedging: Strategies for Managing Foreign Exchange Transaction Exposure, No 162, November 2003

Worthington A, Emergency Finance in Australian Households:

An Empirical Analysis of Capacity and Sources, No 163, November 2003

Worthington C, Debt as a Source of Financial Stress in Australian Households, No 164, November 2003

Robinson M, The Australian Budgeting System: On the Cusp of Change, No 165, November 2003
Lahiri R, Cooperation v/s Non-cooperation in R\&D Competition with Spillovers, No 166, December 2003

Wolff R, Yao Q, \& Tong H, Statistical Tests for Lyapunov Exponents of Deterministic Systems, No 167, December 2003

Wolff R, Barnett A, A Time Domain Test for Some Types of Non-Linearity, No 168 December 2003

\section{LISTING OF DISCUSSION PAPERS - 2004}

Drew M, Veeraraghavan M, Ye M, Do Momentum Strategies Work? Australian Evidence, No 169, January 2004

Drew M, Mallin M, Naughton T, Veeraraghavan M, Equity Premium: - Does it Exist? - Evidence from Germany and United Kingdom, No 170, January 2004

Layton A, Valadkhani A, Measures of National Export Price Volatility Based on the Capital Asset Pricing Model, No 171, January 2004

Drew M, Marsden A, Veeraraghavan M, Small Firm Effect, Liquidity and Security Returns: Australian Evidence, No 172, February 2004

Drew M, Stanford J, Portability of Superannuation Balances, No 173, March 2004

Drew M, Naughton T, Veeraraghavan M Pricing of Equities in China: Evidence from the Shanghai Stock Exchange No 174, May 2004

Valadkhani A, Worthington A, Layton A, An Analysis of the Rising Cost of Education in Australia, No 175, April 2004

Li S, Worthington A, The Relationship Between The Adoption of Internet Banking and Electronic Connectivity: - An International Comparison, No 176, May 2004

Drew M, Marsden A, Veeraraghavan M, Does Idiosyncratic Volatility Matter? - New Zealand Evidence, No 177, May 2004 
Guégan D, How Can We Dane the Concept of Long Memory? - An

Econometric Survey, No 178, April 2004

Clements A, Hurn S, White S, Discretised Non-Linear Filtering for Dynamic Latent Variable Models: with Application to Stochastic Volatility No 179, May 2004

McCarthy S, Ispriani A, An Operating/Economic Exposure Australian Case Study: Foster's Group Limited Discussion Paper No 180, May 2004

Lahiri R, On Skill Heterogeneity and Inflation, Discussion Paper No 181, June 2004 